\title{
The Shear Viscosity in an Anisotropic Unitary Fermi Gas
}

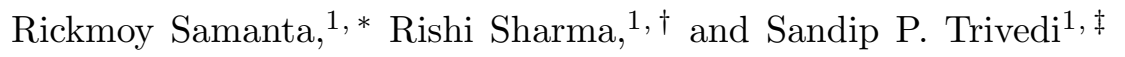 \\ ${ }^{1}$ Department of Theoretical Physics, Tata Institute of Fundamental Research, \\ Homi Bhabha Road, Colaba, Mumbai 400 005, India
}

\begin{abstract}
We consider a system consisting of a strongly interacting, ultracold unitary Fermi gas under harmonic confinement. Our analysis suggests the possibility of experimentally studying, in this system, an anisotropic shear viscosity tensor driven by the anisotropy in the trapping potential. In particular, we suggest that this experimental setup could mimic some features of anisotropic geometries that have recently been studied for strongly coupled field theories which have a gravitational dual. Results using the AdS/CFT correspondence in these theories show that in systems with a background linear potential, certain viscosity components can be made much smaller than the entropy density, parametrically violating the bound proposed by Kovtun, Son and Starinets (KSS). This intuition, along with results from a Boltzmann analysis that we perform, suggests that a violation of the KSS bound can perhaps occur in the unitary Fermi gas system when it is subjected to a suitable anisotropic trapping potential which may be approximated to be linear in a suitable range of parameters. We give a concrete proposal for an experimental setup where an anisotropic shear viscosity tensor may arise. In such situations, it may also be possible to observe a reduction in the spin one component of the shear viscosity from its lowest value observed so far in ultracold Fermi gases. In extreme anisotropic situations, the reduction may be enough to reduce the shear viscosity to entropy ratio below the proposed KSS bound, although this regime is difficult to analyze in a theoretically controlled manner.

PACS numbers: 3.75 Ss, 3.75 Kk, 04.60.Cf, 05.60.-k, 67.85.-d
\end{abstract}

\footnotetext{
*Electronic address: rickmoy@theory.tifr.res.in

${ }^{\dagger}$ Electronic address: rishi@theory.tifr.res.in

${ }^{\ddagger}$ Electronic address: sandip@theory.tifr.res.in
} 


\section{INTRODUCTION}

The calculation of the transport properties of strongly coupled quantum theories is a challenging puzzle of interest to theorists working on a wide range of systems including ultra-cold Fermi gases at unitarity [1, 2], heavy ion collisions [1, 3], and neutron stars [4, 5].

At strong coupling, perturbative expansions fail to give reliable results. Sophisticated MonteCarlo techniques which are used to study such theories non-perturbatively by evaluating pathintegrals in imaginary time, while very successful for calculating equilibrium properties (in the Fermi gas context see Ref. [6] and Refs therein; for heavy ion collisions see Ref. [7] and Refs therein) can not be easily generalized to study transport (in the Fermi gas context see Ref. [8, 9]; for heavy ion collisions see Ref. [10] and Refs therein).

A class of strongly interacting quantum field theories in $d$ dimensions in some limits can be related to weakly coupled theories of gravity (called their dual) in $(d+1)$ dimensions. This correspondence [11] allows us to compute dynamical properties of these theories. These computations have provided many insights into the transport properties of strongly coupled field theories.

In certain limits (large t'Hooft coupling $\lambda$ and large number of "colors" $N_{c}$ ) one can show that for all isotropic theories in $3+1$ dimensions which admit gravity duals, the ratio of shear viscosity $\eta$ to entropy density $s$ is $\frac{\eta}{s}=\frac{1}{4 \pi}$ [12, 13] (we are working in units with $\hbar=1$ and $k_{B}=1$ ). Since weakly coupled theories typically have much larger $\frac{\eta}{s}$, it was conjectured by Kovtun, Son and Starinets (KSS) that $\frac{\eta}{s}$ is bounded from below by $1 /(4 \pi)$. Subsequently it was found that finite $\lambda$ corrections can drive $\frac{\eta}{s}$ below the KSS bound [14-19].

While the theories describing ultra-cold Fermi gases and heavy ion collisions do not have known gravitational duals and controlled calculations are difficult, beautiful experiments have managed to measure the value of $\eta / s$ in the two systems. The value of $\eta / s$ of the quark gluon plasma created in heavy ion collisions, required for hydrodynamic simulations to be consistent with the experimentally measured spectrum of low energy particles (see Ref. [20] for a review), seems to be close to $1 /(4 \pi)$. Remarkably, $\eta / s$ has been measured for ultra-cold fermions at unitarity for a wide range of temperatures and the minimum value (see Refs. [21 23]) is about six times the KSS bound.

On the other hand the shear viscosity tensor for many interesting systems is often anisotropic. For example, it has been suggested that the highly anisotropic initial states in heavy ion collisions (the direction parallel to the collision axes is fundamentally different from the transverse directions) may give rise to anisotropic transport properties [24]. Furthermore, many interesting states of 
matter, eg. spin density waves and spatially modulated phases, are anisotropic. Another possibility, that we shall explore in detail in this paper, is that an externally applied field can pick a particular direction and give rise to anisotropies in the shear viscosity. This possibility has been explored extensively for the case of weakly coupled theories in the presence of a background magnetic field. (See Ref. [25] for a classic treatment, Ref. [26] for applications to heavy ion collisions and Ref. [27] for applications to neutron stars.) The behavior of strongly coupled theories in the presence of an external field is less well explored. With this in mind, anisotropic gravitational backgrounds in field theory have been recently studied using the AdS/CFT correspondence (see [28 36]) and the behavior of the viscosity in some of these anisotropic phases has also been analyzed (see [37, 38. and $[3944]$ ).

The results of Ref. [42] and Ref. [45] for example, indicate that one may obtain parametric violations of the KSS bound in such anisotropic scenarios. This feature arises in a wide variety of examples and seems to be quite general. In particular, for a spatially constant driving force which breaks rotational invariance, it was found that by increasing the strength of the driving force compared to the temperature, the ratio for appropriate components of the shear viscosity to entropy density can be made arbitrarily small, violating the KSS bound.

If this phenomenon also carries over to the unitary Fermi gases, it may be possible to measure these small viscosities in experiments with trapped ultra-cold Fermi gases. For this purpose, it is helpful to consider traps which share the essential features of the systems in Ref. [42, 45] listed at the end of Sec. II of this paper. The goal of this paper is to give a concrete proposal for the trap geometry and parameters where this effect is likely to be seen.

While typical trap potentials are harmonic, [quadratic (Eq. 6) rather than linear in the distance] by using existing results for the thermodynamics of unitary Fermi gases, we show that for a range of temperatures the dominant contribution to the damping of collective modes due to viscosity arises from a narrow region in the trap not near the center, where the trapping potential can be approximately considered as linear. In analogy with Ref. [42, 45] it is desirable to have traps that are highly anisotropic, which can be simulated by taking the trapping frequencies [46] in one of the directions (say $\omega_{z}$ ) to be much larger than the frequencies in the other directions.

We describe two hydrodynamic modes whose dissipation is governed by the components of viscosity which are expected to become small in the anisotropic situation considered here. One of them is known in the literature as the scissor mode which has been well studied for bosonic superfluids at $T=0$ theoretically [47] and has also been experimentally excited in both bosonic [48] and fermionic [49] superfluids. The second mode is a new quasi-stationary solution to the hydrodynamic 
equations. Especially for the scissor mode, we show that for experimentally reasonable values of trap parameters, the damping rate of the mode lies within an experimentally accessible range. It should therefore be possible to study this mode, measure the relevant component of the viscosity and its possible suppression.

To gain some additional understanding of how the anisotropic system might behave, we also make a rough estimate of the viscosity components in the presence of an anisotropic trapping potential using the Boltzmann equation. We find that as the anisotropy increases, due to an increase in the trapping frequency $\omega_{z}$ in one of the directions, some components of the viscosity tensor decrease, compared to their value in the isotropic case.

The outline for the paper is as follows. We review the relevant results [42, 45] for anisotropic theories with gravitational dual in Sec. II and summarize the essential features required in a system to exhibit the suppression of $\eta / s$. Further details on the gravity results is also provided in Appendix A.

Next, we consider the unitary Fermi gas in an anisotropic harmonic trapping potential and describe the two hydrodynamic modes which couple to the small components of the shear viscosity tensor in Sec. III A. In Appendix B 2 and B 3 we show that these two hydrodynamic modes satisfy the equations of superfluid hydrodynamics. Sec. IIIB discusses the energy dissipation due to shear viscosity in these two modes we have studied. In Sec. III C we examine the constraints on the mode amplitudes by demanding validity of fluid mechanics and in Sec. IIID we discuss the damping in the outer regions of the cloud. Next we review the thermodynamics of the system in Sec.IIIE. In Sec. IIIF we give parameter values for traps (the trapping potential, the temperature and the chemical potential at the center of the trap) which are tuned such that the system possesses the required essential features, and show that by measuring the damping rate of fluid modes (described in Sec. III A one can measure the shear viscosity. This section contains some of the key results in the paper. Sec. IV]discusses an analysis in a weakly coupled anisotropic theory using the Boltzmann equation. We conclude our discussion in Sec. V.

The solution of the Boltzmann equation used to estimate the values of the trap potentials for which we expect the corrections to the viscosity to be substantial is given in Appendix $\mathrm{D}$. In Appendix C we compare the modes (discussed in Sec. III A) with the well known breathing modes. 


\section{RESULTS OF SHEAR VISCOSITY FROM GRAVITY}

We briefly review results of computations of shear viscosity in the gravity picture obtained by studying anisotropic blackbranes [42] where the breaking of isotropy is due to an externally applied force which is translationally invariant. The simplest system discussed in Ref. [42] consists of a massless dilaton minimally coupled to gravity, and a cosmological constant. The action is

$$
S=\frac{1}{16 \pi G} \int d^{5} x \sqrt{g}\left[R+12 \Lambda-\frac{1}{2} \partial_{\mu} \phi \partial^{\mu} \phi\right]
$$

where $G$ is Newton's constant in 5 dimensions and $\Lambda$ is a cosmological constant. The dual field theory in the absence of anisotropy is a $3+1$ dimensional conformal field theory. The dilaton profile, linear in the spatial co-ordinate $z$

$$
\phi=\rho z
$$

explicitly breaks the symmetry to $2+1$.

The conservation equation for the stress tensor gets modified as

$$
\partial_{\mu} T^{\mu \nu}=\langle O\rangle \partial^{\nu} \phi
$$

where $O$ is the operator dual to the field $\phi$. The right hand side arises because the varying dilaton results in a driving force on the system. We see that a linear profile results in a constant value for $\partial^{\nu} \phi$ and thus a constant driving force.

Using AdS/CFT one finds [42] that for a system at temperature T, (using the compact notation $\left.\eta_{i j i j}=\eta_{i j}\right) \eta_{x z}=\eta_{y z}$ (which are spin 1 with respect to the surviving Lorentz symmetry) is affected by the background dilaton. In the low anisotropy regime $(\rho / T \ll 1)$ :

$$
\frac{\eta_{x z}}{s}=\frac{1}{4 \pi}-\frac{\rho^{2} \log 2}{16 \pi^{3} T^{2}}+\frac{\left(6-\pi^{2}+54(\log 2)^{2}\right) \rho^{4}}{2304 \pi^{5} T^{4}}+\mathcal{O}\left[\left(\frac{\rho}{T}\right)^{6}\right]
$$

The correction to the zero anisotropy result, the KSS bound $\frac{\eta_{x z}}{s}=\frac{1}{4 \pi}$, is proportional to $\frac{(\nabla \phi)^{2}}{T^{2}}$ where $\nabla \phi=\rho \hat{z}$ is the driving force and $1 / T$ is the microscopic length scale in the system.

In extreme anisotropy $(\rho / T \gg 1)$,

$$
\eta_{x z} / s \rightarrow(1 / 4 \pi)\left(32 \pi^{2} T^{2} / 3 \rho^{2}\right)
$$


and hence becomes parametrically small [42]. But this domain will not be physically accessible in the cold atom systems.

In contrast the $\eta_{x y}$ component (which couples to a spin 2 metric perturbation) was found to be unchanged from its value in the isotropic case, $\frac{\eta_{x y}}{s}=\frac{1}{4 \pi}$.

Parametric reduction of the spin 1 components of $\eta / s$ has been found for a variety of strongly coupled theories with a gravitational dual [37, 45]. Motivated by the generality of the above results, (see [45]) in the gravity side, we may hope to find parametrically suppressed viscosities compared to the KSS bound in systems where the following basic requirements are met.

1. The system is strongly interacting and in the absence of anisotropy have a viscosity close to the KSS bound.

2. The equations of hydrodynamics for the system admits modes sensitive to the spin one viscosity components as described above and in Ref. [42, 45].

3. Sufficient anisotropy needs to be introduced in the system (say in the $z$ direction with rotational symmetry preserved along the $x-y$ plane), such that these spin one components of the viscosity, when measured in units of the entropy density, show an experimentally measurable decreasing tendency from its lowest value observed so far in ultracold Fermi gases.

4. The force responsible for breaking of isotropy is approximately spatially constant.

5. The velocity gradients are small enough (compared to say the inverse mean free path) ensuring that hydrodynamics is the appropriate effective theory to describe the system.

In the next section (Sec. III) we explore a system of trapped ultra-cold Fermi gases, chosen so as to explore anisotropic fluid dynamics. While some of the details of this system are different from the systems with dual gravitational theories discussed above, it is possible to choose a set of parameters such that the system has the five features listed above. It can therefore be used to explore the behavior of the viscosity in the anisotropic regime.

While gravitational duals for the ultra-cold Fermi gases are not yet known and hence we can not calculate the anisotropic viscosity coefficients in this strongly coupled system, if the main feature that $\eta_{x z}$ is smaller than the KSS bound holds true for these, one could potentially measure this phenomenon in experiments. 


\section{ANISOTROPIC VISCOSITY IN TRAPPED ANISOTROPIC FERMI GASES}

Trapped ultra-cold Fermi gas with their scattering length tuned to be near the unitarity limit 46. 50], are strongly interacting systems for which $\eta / s[21-23$, was measured to be close to the KSS bound $1 /(4 \pi)$. In this section we shall explore the properties of this system, when it is placed in an anisotropic trap. We identify suitable hydrodynamic modes which probe the viscosity component expected to be suppressed due to the potential in a highly anisotropic harmonic trap and find that for reasonable choices of parameters the five criterion referred to above, (see Sec.II), can be met in these modes. This leads us to suggest that an anisotropic shear viscosity can arise in such systems and appropriate components of the viscosity may show a reduction from the isotropic values in an experimentally accessible way.

One method [23] to measure the viscosity is by starting with an initial state where the fluid is trapped in an anisotropic harmonic trap. On removing the trapping potential, the fluid experiences elliptic flow and the extent of the flow is related to the initial anisotropy and the viscosity. The relevant bulk viscosity of the system vanishes [51, 52, which allows one to cleanly extract the shear viscosity. Note that even though the initial state of the fluid is anisotropic, the experiment does not probe anisotropic shear viscosities: after the trap potential is removed, the viscosity tensor at any point is isotropic.

An alternative technique is to measure the damping rate of breathing modes [21, 22] which is related to the loss of energy due to the viscosity. The experiments we propose in this paper use this alternative technique and propose to measure the relevant component of the shear viscosity by measuring the damping of appropriate hydrodynamic modes.

The unitary Fermi gas system we consider here shares important features with the gravitational system described in Sec. III. The role of a linear potential was emphasized in Sec. II. While such a linear potential cannot arise in the trapped fermion system we consider, we shall see below that if we choose the velocity profile and the trap parameters carefully, the dominant contribution to shear viscosity comes from a region of the trap where the confining force is approximately constant: satisfying the fourth criterion listed in Sec. II.

The system we consider consists of an ultra-cold Fermi gas under harmonic confinement described by the potential

$$
\phi(\mathbf{r})=\sum_{i} \frac{1}{2} m \omega_{i}^{2} x_{i}^{2}
$$



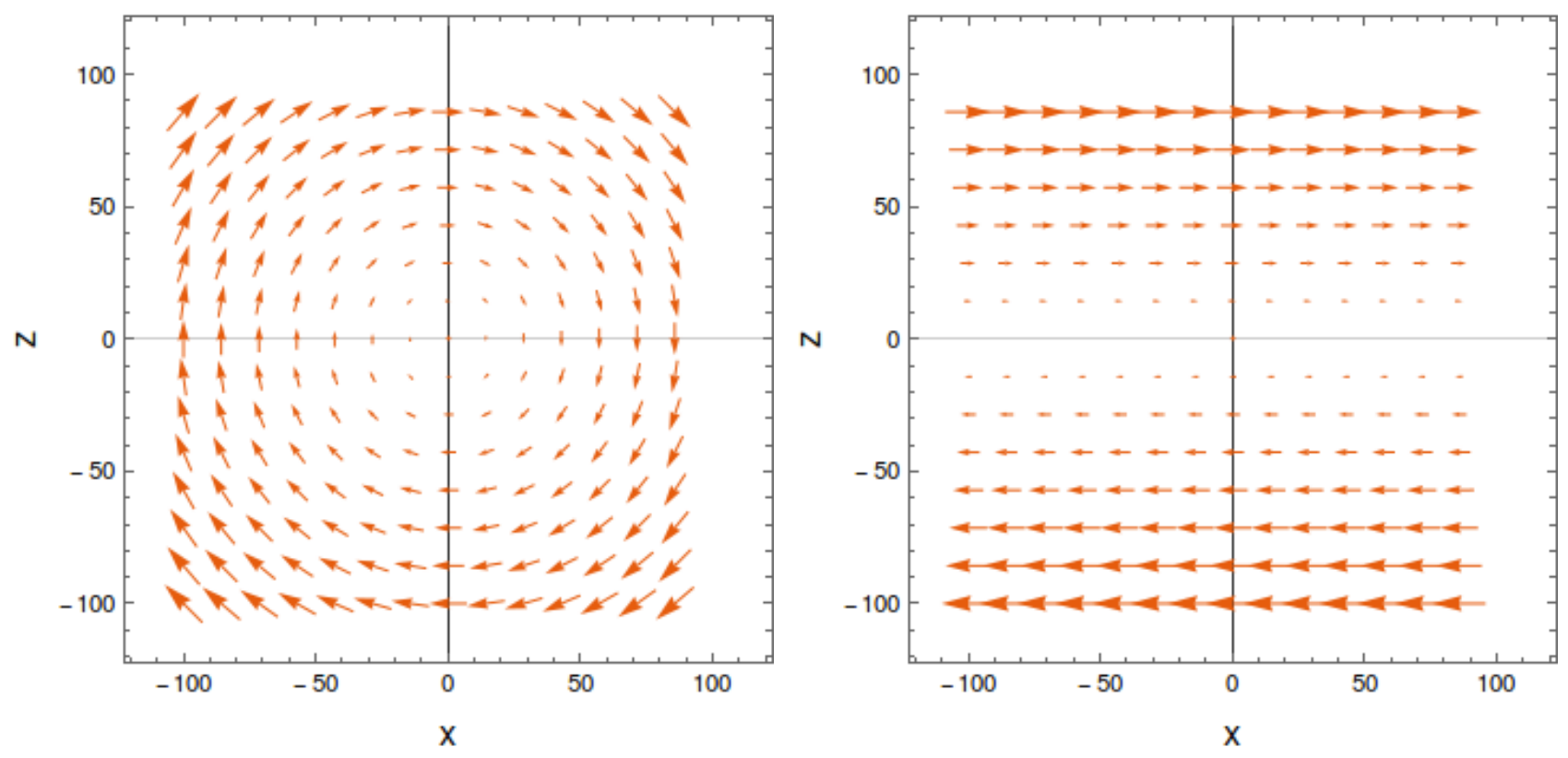

FIG. 1: (Arbitrary units for coordinates) The flow profile in the $x-z$ plane for the Elliptic mode, ie. $\mathbf{v}=z \hat{x}-x \hat{z}$ (left panel, corresponding to $\omega_{x} / \omega_{z}=1$ in Eq. 8) and $\mathbf{v}=z \hat{x}-0.001 x \hat{z}$ (right panel, corresponding to $\omega_{x} / \omega_{z}=0.03$ in Eq. 8).

where $i$ runs over $x, y, z$ and $m$ denotes the mass of the fermionic species. The trap is anisotropic if $\omega_{i}$ 's are unequal. For example, $\omega_{z} \gg \omega_{x}, \omega_{y}$ gives rise to a pancake like trap: thin in the $z$ direction. This can lead to an anisotropic shear viscosity tensor as described in Sec. IV] The potential gradient in the $x$ and $y$ directions is small in most of the trap.

This section is organized as follows. After a general discussion we describe the two modes of interest (referred to as the Elliptic mode and the Scissor mode) in subsection III A. The equations of superfluid hydrodynamics are described in Appendix B1, following which, in Appendix B2 and B3 respectively we show that the Scissor mode and the Elliptic mode satisfy these equations. The fluid flow profile in the Elliptic mode is similar to that shown in Fig. 8, a velocity in the $x$ direction with a gradient in the $z$ direction. The scissor mode is well known in the literature. In subsection III B we show that the dissipation of energy in the two modes of interest is determined by the relevant components of the viscosity tensor (the spin 1 components described in the previous section). In Subsection III C we find a constraint on the magnitude of the velocity for the two modes by demanding the validity of fluid mechanics. The thermodynamics of the system is discussed in subsection IIIE, Finally in subsection IIIF we bring this understanding together and show that for reasonable values of parameters the required criterion listed in Sec. II can indeed be met. 


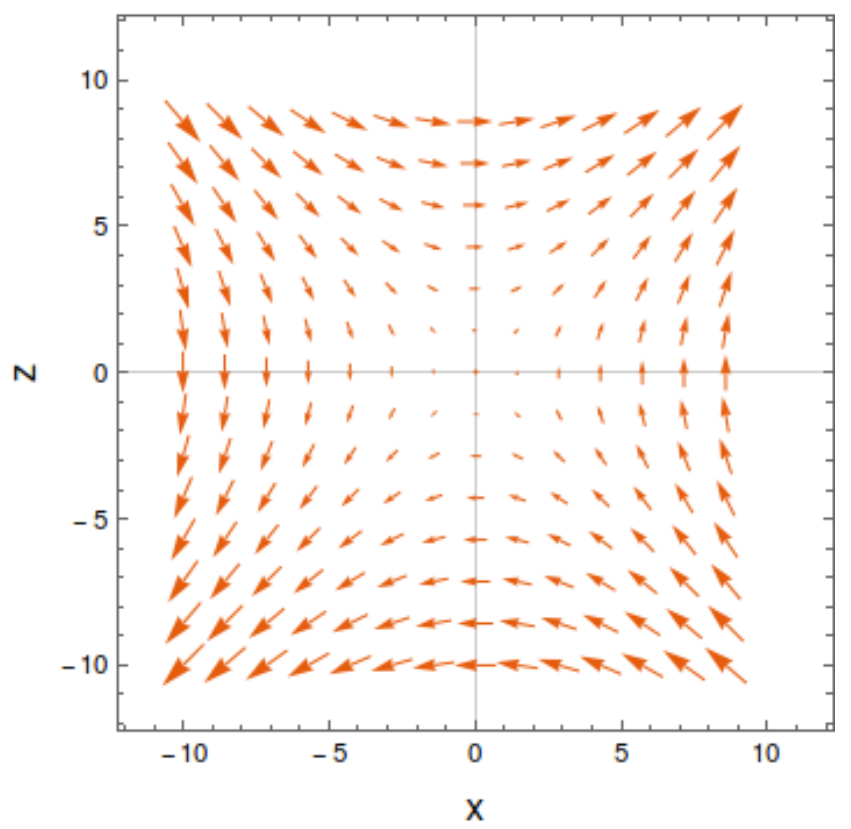

FIG. 2: (Arbitrary units for coordinates) The flow profile in the $x-z$ plane at time $t=0$ for the Scissor mode, ie. $v=z \hat{x}+x \hat{z}$ (Eq. 9)

\section{A. Choice of Velocity Profile}

Here we first describe the two modes of interest which arise as solutions to the equations of ideal superfluid hydrodynamics. Each of these modes is characterized by the superfluid and the normal components, which we denote by $\mathbf{v}_{s}$ and $\mathbf{v}_{n}$ respectively.

The first mode, which we call the Elliptic mode has $\mathbf{v}_{s}=0$ and $\mathbf{v}_{n}=\mathbf{v}$ given by

$$
\mathbf{v}=e^{i \omega t}\left(\alpha_{x} z \hat{x}+\alpha_{z} x \hat{z}\right)
$$

with the following relations:

$$
\text { Elliptic mode : } \omega=0, \alpha_{z}=-\frac{\omega_{x}^{2}}{\omega_{z}^{2}} \alpha_{x}
$$

The other mode of interest, denoted by the Scissor mode, has $\mathbf{v}_{s}=\mathbf{v}_{n}=\mathbf{v}$ given by Eq. 7 with

Scissor mode : $\omega=\sqrt{\omega_{x}^{2}+\omega_{z}^{2}}, \alpha_{z}=\alpha_{x}$.

From the right panel in Fig. 1 we see that in the high anisotropy limit $\omega_{z} \gg \omega_{x}, \alpha_{z} \rightarrow 0$ for the Elliptic mode, and hence we recover a flow profile similar to that considered in [42] (shown 
in Fig. 8); namely a time independent (in the limit of small viscosity) velocity $(\mathbf{v} \propto z \hat{x})$ linearly increasing with the coordinate in the direction of the gradient of the external potential $(z)$, pointing $(\hat{x})$ in the direction perpendicular to the gradient of the external potential (neglecting $\omega_{x}, \omega_{y}$. The gradient is in the $\hat{z}$ direction). To the best of our knowledge, the Elliptic mode has not been studied in ultra-cold gas experiments. The scissors mode which has been studied extensively (for example see Refs. [47-49]).

\section{B. Energy dissipation due to viscosity}

The energy dissipated due to viscosity is given by

$$
\dot{E}_{\text {kinetic }}=-\frac{1}{2} \int d^{3} \mathbf{r} \eta_{i j i j}(\mathbf{r})\left(\partial_{i} v_{j}+\partial_{j} v_{i}-\frac{2}{3} \delta_{i j} \partial_{k} v_{k}\right)^{2}-\int d^{3} \mathbf{r} \zeta(\mathbf{r})\left(\partial_{i} v_{i}\right)^{2}
$$

where $\eta_{i j i j} \equiv \eta_{i j}$ is the relevant component of the shear viscosity and $\zeta$ is the bulk viscosity. We note that for our chosen velocity profiles, the bulk viscosity contribution vanishes. Also in the traps we will consider, the temperature $\mathrm{T}$ is constant throughout the trap. Hence we also ignored contributions from thermal conductivity.

Thus,

$$
\dot{E}_{\text {kinetic }}=-\int d^{3} \mathbf{r} \eta_{x z}(\mathbf{r}) \alpha_{x}^{2}\left(1-\frac{\omega_{x}^{2}}{\omega_{z}^{2}}\right)^{2}
$$

is the energy dissipation rate for the Elliptic mode, where we have simply written $\eta_{x z x z}$ as $\eta_{x z}$.

The energy dissipated per unit cycle for the oscillatory time dependent scissor mode is

$$
\dot{E}_{\text {kinetic }}=-2 \int d^{3} \mathbf{r} \eta_{x z}(\mathbf{r}) \alpha_{x}^{2}
$$

\section{Validity of hydrodynamics}

One expects that hydrodynamics is a valid description of the system as long as the viscous correction to the stress tensor is small compared to its value in an ideal fluid (for eg. see Ref. [53] or Sec. 10.3.4 in Ref. [54]).

For the Elliptic mode the contribution to the stress energy tensor from viscosity is

$$
\eta_{x z} \frac{1}{2}\left(\alpha_{x}+\alpha_{z}\right) \approx \eta_{x z} \frac{1}{2}\left(\alpha_{x}\right)
$$


where we have assumed $\omega_{z} \gg \omega_{x, y}$ and neglected the contribution from $\alpha_{z}$ (see Eq. 8).

For the Scissor mode the magnitude of the contribution to the stress energy tensor from viscosity is

$$
\eta_{x z} \frac{1}{2}\left(\alpha_{x}+\alpha_{z}\right)=\eta_{x z}\left(\alpha_{x}\right)
$$

where we have $\alpha_{z}=\alpha_{x}$ for the Scissor mode.

At any point $\mathbf{r}$, hydrodynamics is expected to be valid if the viscosity contribution is smaller than the pressure $P(\mathbf{r})$,

$$
\alpha_{x} \eta_{x z}(\mathbf{r}) \ll P(\mathbf{r})
$$

In the outer edges of the trap the pressure becomes small while $\eta$ tends to a constant [55, 58] and Eq. 15 is necessarily violated regardless of how small $\alpha_{x}$ is chosen. The contribution of this region to the total energy loss is typically small however. (Note that the expression Eq. 10 can not be used to evaluate the energy loss if Eq. 15 is not satisfied [57].) What we desire is that hydrodynamics should be a good theory in the region where the energy loss is substantial. When we consider specific numerical values for the parameters of the trap in Subsection IIIF, we will identify a point $\mathbf{r}_{\max }$ close to the edge of the trap, such that the integral Eq. 10 receives most of its contribution for $r<\mathbf{r}_{\max }$.

We can then define $\alpha_{x}^{\max }$ by the condition that for this amplitude the viscosity contribution to the stress energy tensor is equal to the pressure at the point $\mathbf{r}_{\max }$

$$
\alpha_{x}^{\max }=\frac{P\left(\mathbf{r}_{\max }\right)}{\eta_{x z}\left(\mathbf{r}_{\max }\right)}
$$

For $\alpha_{x}<\alpha_{x}^{\max }$ hydrodynamics is valid in the region of interest. This constraint limits how large $\alpha_{x}$ and consequently $\dot{E}_{\text {kinetic }}$ can be. As long as this dominates over other processes of energy loss (interaction with the environment) this damping can be measured. In Table. III in Sec. IIIF we show this numerical limit for the traps described in that Section. 


\section{The outer core}

It has been noted that a naive application of hydrodynamics at the outer region of the trap where the density of the atoms is very low leads to an unphysical result. Since the shear viscosity in the ultra-dilute regime has the form $\eta \sim(m k T)^{3 / 2},(m$ is the mass, $k$ is the Boltzmann's constant and $T$ is the temperature) the contribution from the tail (or the outer cloud) is independent of the density, and hence is divergent [[55,62]]. The unphysical result arises because in the outer part of the trap collisions are rare and hydrodynamics breaks down. In fact the better approximation in this region is assuming that atom dynamics in this ultra-dilute region is collisionless and hence does not contribute significantly to damping.

Here we use a simple procedure to take this physics into account. We only consider traps where the chemical potential at the center is positive and cutoff the damping contribution from the outer cloud by integrating the viscosity contribution only from the center of the trap up to $\mathbf{r}_{\max }$ which is defined as the surface where $\mu-V\left(\mathbf{r}_{\max }\right)=T$. We have checked that changing $r_{\max }$ by a little (for example by choosing a slightly larger $r_{\max }^{0}$ by using the condition $\mu-V\left(r_{\max }^{0}\right)=0$ ) gives similar results for the damping rates. Similar prescriptions have been followed previously by [22, 23] (see [62] for an overview).

One can also perform a more careful estimate of the contribution from the outer cloud. To be concrete, let us consider the scissor mode. We follow the procedure described in Ref. [59] which solves the Boltzmann equation in the dilute regime, rather than assuming that hydrodynamics is accurate in this region. Their important result is that for the scissor mode ${ }^{1}$ the energy loss rate in the dilute regime can be written as the integral over $\eta$ divided by a suppression factor that increases exponentially as a function of the trapping potential. More precisely,

$$
\left.\left\langle\dot{E}_{\text {kinetic }}\right\rangle\right|_{\text {oc }}=-2 \alpha^{2} \int_{\mathbf{r}>\mathbf{r}_{\max }} d^{3} \mathbf{r} \frac{\eta}{1+\omega^{2} \tau_{\eta}^{2}(\mathbf{r})}
$$

where in the dilute regime (or the "classical limit")

$$
\tau_{\eta}(\mathbf{r})=\frac{4.17}{N \bar{\omega}}\left(\frac{k T}{\hbar \bar{\omega}}\right)^{2} e^{V(\mathbf{r}) / k T}
$$

\footnotetext{
${ }^{1}$ Let us also note that the scissor mode is excited in the $x-y$ plane in Ref. [59]. We have taken care of this fact in our calculations and comparisons.
} 
and the viscosity $\eta$ is given by

$$
\eta=\frac{15}{32 \sqrt{\pi}} \frac{(m k T)^{3 / 2}}{\hbar^{2}}
$$

The scissor mode frequency is given by,

$$
\omega=\sqrt{\omega_{x}^{2}+\omega_{z}^{2}}
$$

and the geometric mean $\bar{\omega}=\left(\omega_{x} \omega_{y} \omega_{z}\right)^{\frac{1}{3}}$.

The integral Eq. 17 is convergent because of the exponential increase in the relaxation time $\tau_{\eta}(\mathbf{r})$ even if we take the upper limit of the integral to $\infty$ but for the numerical evaluation we take the upper limit of the $x$-integration to be $x_{\max }+L$, for the $y$-integration to be $y_{\max }+L$, and $z$-integration to be $z_{\max }+L$ with $L \gg\left|\mathbf{r}_{\max }\right|$.

At the core of the trap hydrodynamics is a good approximation (unless $T \ll T_{c}$ where the superfluid phonons can move out of equilibrium). This is a crucial point because Boltzmann transport is not a valid approximation at the core where the density of atoms is high. As we explained in the last section, as long as $\alpha_{x}=\alpha_{z}=\alpha<\alpha_{x}^{\max }$, hydrodynamics is a good approximation and the local contribution from the viscosity to the stress energy tensor

$$
\alpha \eta(\mathbf{r})
$$

is smaller than the pressure

$$
P(\mathbf{r})
$$

for $\mathbf{r}<\mathbf{r}_{\max }$. Therefore, using hydrodynamics to evaluate the damping contribution from the core, we get

$$
\left.\left\langle\dot{E}_{\text {kinetic }}\right\rangle\right|_{\mathrm{c}}=-2 \alpha^{2} \int_{\mathbf{r}<\mathbf{r}_{\max }} d^{3} \mathbf{r} \eta(\mathbf{r})
$$

where the local value of $\eta(\mathbf{r})$ is calculated using the data for $\eta$ from [23]. The integration is performed over $x<x_{\max }, y<y_{\max }$ and $z<z_{\max }$. This approximates the actual ellipsoidal region with a rectangular shape, but we see that this will not change the results substantially since the contribution from the outer cloud is small. 


\begin{tabular}{|c|c|c|}
\hline$T$ & $\Gamma_{\mathrm{c}}\left(s^{-1}\right)$ & $\Gamma_{\mathrm{oc}}\left(s^{-1}\right)$ \\
\hline $4 T_{c} / 5$ & 23.03 & 0.0044 \\
$2 T_{c} / 3$ & 18.32 & 0.00009 \\
$4 T_{c} / 7$ & 14.6 & $2.14 \times 10^{-6}$ \\
$T_{c} / 2$ & 11.86 & $4.69 \times 10^{-8}$ \\
\hline
\end{tabular}

TABLE I: Comparison of contributions to the damping rates for the scissor mode from the core [Г(c) Eq. 27], and the outer core $[\Gamma(\mathrm{oc}) \mathrm{Eq} \cdot 26$ for the trap parameters we will explore in our paper.

The amplitude decay rate is given by

$$
\Gamma=\frac{\left|\left\langle\dot{E}_{\text {kinetic }}\right\rangle\right|}{2\langle E\rangle}
$$

$\langle E\rangle$ is the total mechanical energy averaged over a cycle,

$$
\begin{aligned}
\langle E\rangle & =\frac{1}{2} \int d^{3} \operatorname{rmn}(\mathbf{r})|v|^{2}(\mathbf{r}) \\
& =\frac{1}{2} m \alpha^{2} \int d^{3} r m n(\mathbf{r})\left(z^{2}+x^{2}\right),
\end{aligned}
$$

where $v=\alpha e^{i \sqrt{\omega_{x}^{2}+\omega_{z}^{2}} t}(z \hat{x}+x \hat{z})$. In Eq. 27, $\alpha^{2}$ cancels out and we only need $n(\mathbf{r})$ which is obtained from experiments as explained in detail in SecIIIE.

The damping rate contribution from the outer cloud is given by

$$
\Gamma_{\mathrm{oc}}=\frac{\left|\left\langle\dot{E}_{\mathrm{kinetic}}\right\rangle\right|_{\mathrm{oc}}}{2\langle E\rangle}
$$

and the contribution from the core is given by

$$
\Gamma_{\mathrm{c}}=\frac{\left|\left\langle\dot{E}_{\mathrm{kinetic}}\right\rangle\right|_{\mathrm{c}}}{2\langle E\rangle}
$$

and the total damping rate Eq. 24 is the sum of the two.

In Table. I], for the representative trap parameters which we will be considering later ( $\omega_{z}=2 \pi \times 10^{4} \mathrm{rads} / \mathrm{s}, \omega_{x}=\omega_{y}=2 \pi \times 385 \mathrm{rads} / \mathrm{s}$ and $\mu=10 \mu \mathrm{K}$ and $T / T_{c}$ values as given in the table), we present the comparison of the contribution to damping from the outer cloud and the core in Table. I. We see that the damping contribution from the outer cloud is small, especially for the low temperatures, justifying our approach. A direct comparison using our technique (where we cut off the integral for $\dot{E}_{\text {kinetic }}$ at the point of the trap where hydrodynamics breaks down) can only be made for the lowest temperature $\left(T / T_{F}=0.1\right.$ ) of Ref. [49]. Our calculations (using the 

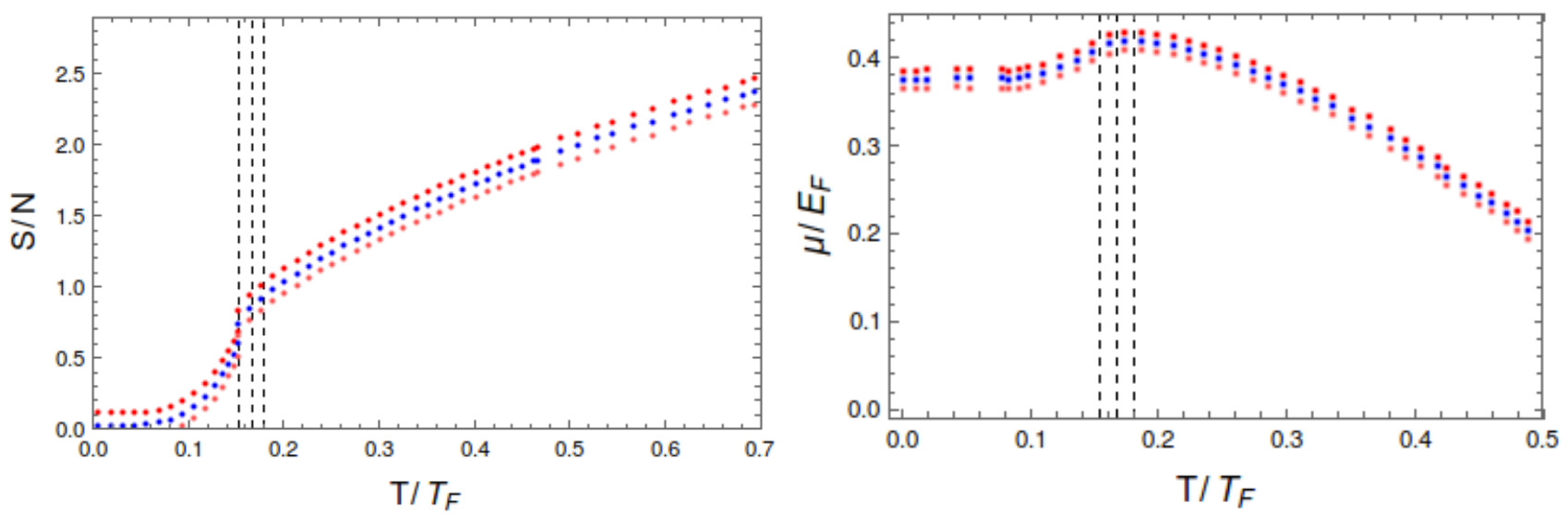

FIG. 3: (Color online) Data of $\frac{S}{N}$ as a function of $T / T_{F}$ (left panel) and $\mu / E_{F}$ versus $T / T_{F}$ (right panel) from Ref. 63. The central curves (blue online) correspond to the central values and the band gives an error estimate (Ref. 63]). The band denoted by the dashed vertical lines corresponds to the phase transition between the normal and the superfluid phase. The error bands represent the maximum error chosen from a set of representative error bars given in Ref. [63].

trap parameters of [49]) give a damping rate of $250 \mathrm{~s}^{-1}$ which agrees with experiments $(255 \pm 40$ $s^{-1},[49$ ). This is a non-trivial check of our methodology and gives us confidence in our approach in this regime.

\section{E. Thermodynamics}

The evaluation of the energy loss from Eq. 11 and Eq. 12 requires the viscosity $\eta$ as a function of the position $\mathbf{r}$ in the trap. In the highly anisotropic traps we are considering the viscosity is actually a tensor and the different components of the shear viscosity can acquire different values, in contrast with the isotropic case. For the modes of interest, Eq. 7 we need to determine the behavior of the component $\left(\eta_{x z}\right)$.

To get a first estimate of the region of the trap which gives a dominant contribution to the integral in Eq. 10, we use the local density approximation (LDA) and estimate the resulting viscosity. More specifically, we assume in this approximation that thermodynamic variables like the number density $n$, the entropy density $s$ depend only on the local value of $T$ and $\mu$. The viscosity is also then taken to be given by these local values of $T, \mu$, neglecting any effects of anisotropy which could make the different components of the tensor take different values.

The effect of anisotropy on the viscosity tensor are estimated using Eq. 65, in a following section (Sec. IV). While we cannot reliably compute them, the key point of our analysis here is that they may be experimentally measured and could lie below the KSS bound. 

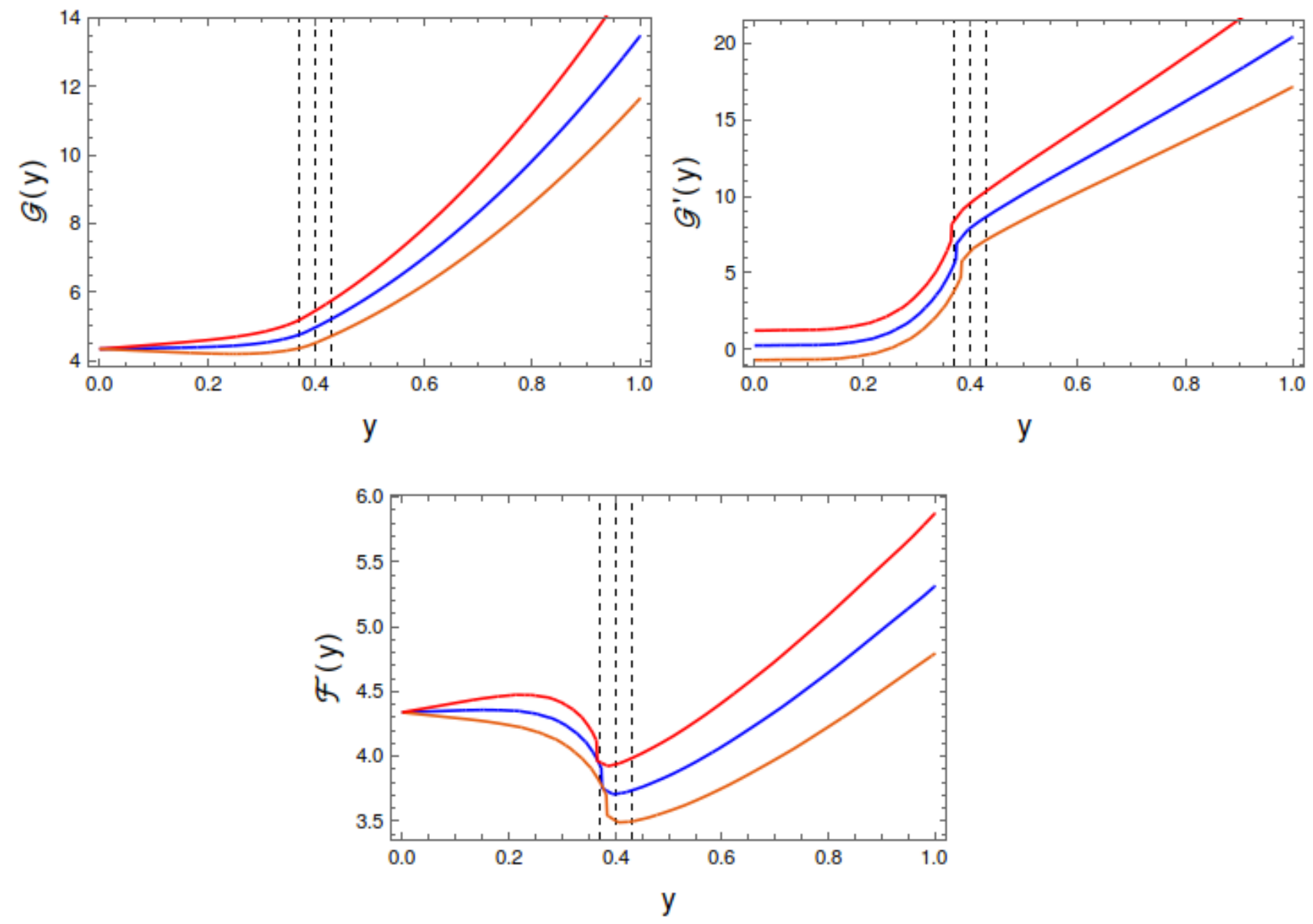

FIG. 4: (Color online) The thermodynamic function $\mathcal{G}$ (top left panel) and its derivative (top right panel) as a function of $\frac{T}{\mu}$. The lower panel shows $\mathcal{F}$. These dimensionless functions are defined in Eq. 28. The error bands follow from the error bands in Fig. 3

To apply the LDA approximation mentioned above, we start first by considering a homogeneous system characterized by temperature $T, \mu$ and review the behavior of the thermodynamical parameters and the viscosity as a function of these parameters. This is covered in this subsection. In the presence of the trap $\mu$ varies in the equilibrium configuration. The effects of the trap, in this approximation, are then incorporated by using the resulting local value for $\mu$ and $T$ in the behavior for the homogeneous case. The next subsection will then incorporate the effects of the trap.

In certain thermodynamic regimes, the viscosity of a uniform unitary Fermi gas can be computed in a controlled manner. At temperatures much smaller than the chemical potential, transport is dominated by the Goldstone mode associated with superfluidity and the viscosity can be computed by solving the Boltzmann transport equations [64]. At temperatures large compared to the chemical potential, the density of fermions is small and a kinetic estimate of the viscosity, $\eta=$ const. $\times$ 

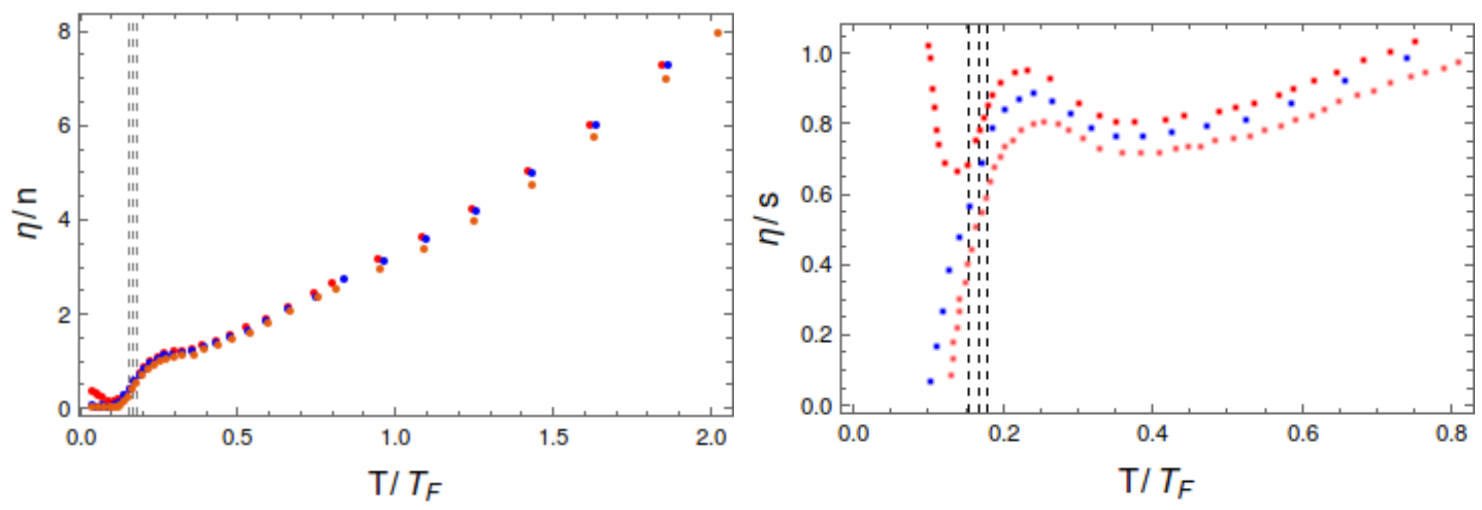

FIG. 5: (Color online) The left panel shows $\frac{\eta}{n}$ versus $T / T_{F}$ from Figs. 2 and 3 of [23]. The right panel shows $\frac{\eta}{s}$ versus $T / T_{F}$ from Fig. 5 of [23].

$(m T)^{3 / 2}$, is adequate [55 57]. But we shall see that the largest contribution to damping arises from the regime where $T$ and $\mu$ are comparable, and a theoretical evaluation of the viscosity is difficult. Monte Carlo [8, 9] methods, microscopic approaches [65], and $T$-matrix techniques [66] have been used to calculate the viscosity in this regime but presently the best estimate for the viscosity in this intermediate regime comes from experiments.

In Refs. 21, 22, $\eta / s$ was measured for the first time. Recently, this measurement was refined in Ref. 23] and the result for the dimensionless ratio $\eta / n$ was measured for a wide range of $T / \mu$, which we show in Fig. 5. Therefore, to obtain the LDA value of the viscosity, we just need $n(\mu, T)$.

In the next few paragraphs we describe how to obtain $n(\mu, T)$ using the scaling properties of the unitary Fermi gas. With that understanding at hand we will then return to a discussion of how to obtain the viscosity in the approximation described above. In the unitary Fermi gas, the chemical potential $\mu$ and the temperature $T$ are the only energy scales in the problem. Therefore, we can express various thermodynamic quantities as a function of the dimensionless quantity $y=T / \mu$ multiplied by an appropriate dimensionful function of only one of the two variables. Following [21] we write,

$$
\begin{aligned}
n(\mu, T) & =n_{f}(\mu) \mathcal{F}(y), \\
s(\mu, T) & =\frac{2}{5} n_{f}(\mu) \mathcal{G}^{\prime}(y),
\end{aligned}
$$

where $n$ is the number density, $s$ is the entropy density, and $\mathcal{F}(y)=\mathcal{G}(y)-2$ y $\mathcal{G}^{\prime}(y) / 5, n_{f}(\mu)=$ $\frac{1}{3 \pi^{2}}(2 m \mu)^{\frac{3}{2}}$ is the number density of a free Fermi gas. Therefore one can compute the desired thermodynamic quantities if the function $\mathcal{G}(y)$ is known. For example, one can write the pressure 


$$
P(\mu, T)=\frac{2}{5} \mu n_{f}(\mu) \mathcal{G}(y)
$$

In the following discussion, we use the usual definitions

$$
k_{F}=\left(3 \pi^{2} n\right)^{1 / 3}, E_{F}=\frac{k_{F}^{2}}{2 m}, T_{F}=E_{F} / k_{B}, v_{F}=\frac{k_{F}}{m}
$$

At low temperatures $\left(\frac{T}{T_{F}} \lesssim 0.6\right)$ we use the $\frac{S}{N}$ data from Fig. $3(b)$ of Ref. [63] to obtain $\mathcal{G}(y)$. Data from two graphs obtained from Ref. 63] are shown here in the two panels of Fig. 3 for convenience. The left panel shows $S / N=s / n$ as a function of $T / T_{F}$ and the right panel shows $\mu / E_{F}$ as a function of $T / T_{F}$.

In order to solve Eq. 28 we need to get $\frac{S}{N}$ as a function of $y$. We use Fig. 3(a) of Ref. 63] to convert the $\frac{S}{N}$ data in terms of $y=\frac{T}{\mu}$ rather than $\frac{T}{T_{F}}$. We obtain the function $\mathcal{G}(y)$ by numerically solving Eq. 28 , subject to the boundary condition $\mathcal{G}(0)=1 / \xi^{3 / 2}$ at $T=0$. We use $\xi=0.376 \pm 0.0075$. (The value of $\xi$ quoted here is from [63. Various theoretical calculations can be found in [6, 67-72].) Fig. 4 shows the numerically extracted function $\mathcal{G}$, its first derivative and the function $\mathcal{F}$. In Fig. 4 and the rest of the figures, the band denoted by the dashed vertical lines corresponds to the phase transition between the normal and the superfluid phase.

The data in Ref. 63] stops at $T / T_{F} \approx 0.6$. For higher temperatures the density is small and as far as thermodynamics is concerned, we can model the system as a gas of weakly interacting fermions with a self energy correction in the chemical potential associated with self interactions in the normal phase. Therefore $n$ and $s$ have the same form as in a Fermi gas, (Ref. [73])

$$
\begin{aligned}
n_{\text {norm }} & =-g(m T)^{\frac{3}{2}} \frac{\operatorname{PolyLog}\left(\frac{3}{2},-e^{\frac{\mu}{T}}\right)}{2 \sqrt{2} \pi^{3 / 2}} \\
s_{\text {norm }} & =\frac{\sqrt{T}\left(2 \mu \operatorname{PolyLog}\left(\frac{3}{2},-e^{\frac{\mu}{T}}\right)-5 T \text { PolyLog }\left(\frac{5}{2},-e^{\frac{\mu}{T}}\right)\right)}{2 \sqrt{2} \pi^{3 / 2}},
\end{aligned}
$$

where $n_{\text {norm }}, s_{\text {norm }}$ denote the number density and entropy in the normal phase, $g=2$ is the energy level degeneracy, and $\mu$ with self energy corrections is replaced by $\mu-\frac{3^{2 / 3} n^{2 / 3} \pi^{4 / 3}\left(\xi_{n}-1\right)}{2 m}$. Fitting to high temperature data gives $\xi_{n} \approx 0.45[63$. This description works well all the way down to temperatures $T / T_{F} \gtrsim 0.5$ or equivalently $\frac{T}{\mu} \gtrsim 3.2$ as one can check by comparing the values of $S / N$ as a function of $T / T_{F}$ in this approximation with the results from [73]. These results match 

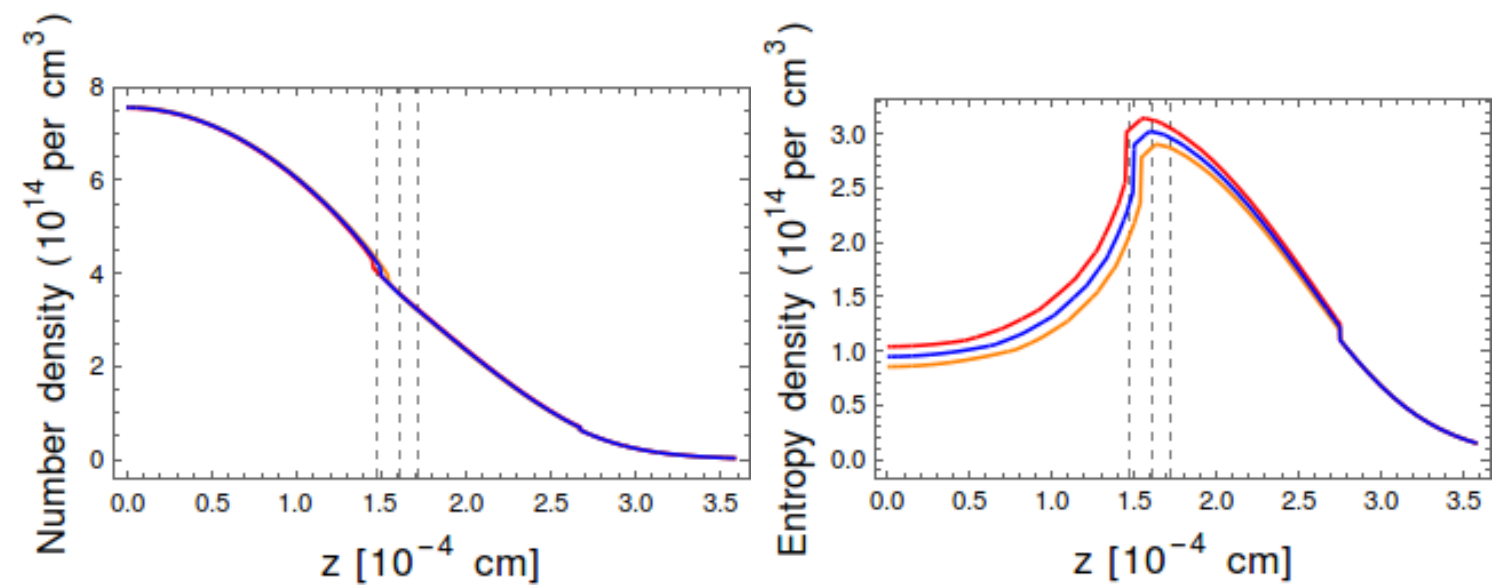

FIG. 6: (Color online) Variation of number density (left panel) and the entropy density (right panel) with respect to $z$ for $T=\frac{2 T_{c}}{3}$ at $\omega_{z}=2 \pi \times 10^{4} \mathrm{rads} / \mathrm{s}$ with chemical potential at the trap center $10 \mu \mathrm{K}$. The vertical lines denote the band in $z$ where $T=(0.4 \pm 0.03)(\mu-\phi(z))$ (Eq. 33).

smoothly to the low temperature measurements in Ref. [63]. Therefore for $\frac{T}{\mu}>3.2$ we use Eq. 31 to compute the thermodynamics.

Now that we have understood how to obtain $n(T, \mu)$ we can return to our discussion of the viscosity. To evaluate $\eta$ at a given $\mu$ and $T$ we simply multiply $\frac{\eta}{n}$ from Fig. 3 of Ref. [23] (shown here in the left panel of Fig. 5) with the number density that can be found using Eq. 28. One could alternatively multiply $\frac{\eta}{s}$ from Fig. 5 of Ref. [23] (shown here in the right panel of Fig. 5) with the entropy that can be found using Eq. 28. The former works better because of the smaller error bars.

As we shall see in the next section when we describe the fermions in a trap, the dominant contribution to the energy loss arises from the region in the trap where $T / \mu$ is about 0.54 . This is just above the critical temperature $T_{c}$ given by the relation

$$
T_{c} / T_{F}=0.167 \pm 0.013
$$

or equivalently

$$
\frac{T_{c}}{\mu}=0.4 \pm 0.03
$$

From the right panel of Fig. 5 we see that just above $\frac{T_{c}}{\mu} \approx 0.4, \eta / s \approx 0.7 \approx 8\left(\frac{1}{4 \pi}\right)$. This fact will be relevant in the next section. 


\section{F. Results for the trap}

Having understood the thermodynamics in the absence of the trap, we now turn to incorporating the trap potential in the discussion. We first use the LDA approximation to calculate how thermodynamic quantities like $s, n$ etc. vary along the trap. It turns out that on starting at the center of the trap at a sufficiently low temperature, the entropy density has a peak, $z_{0}$, close to the point where the superfluid-normal transition occurs. In turn, this leads to the viscosity and damping effects for the fluid modes of interest receiving their contribution from a region close to the peak and with a width, $\delta z$ that can be made narrow, $\delta z / z_{0}<1$. Finally, in this subsection we examine the resulting behavior of the system for a range of reasonable values of parameters and show that the five conditions listed at the end of Section $\amalg$ can be met. It turns out that both the time scales for energy loss, and the magnitude of the total energy, lie in the range of experimentally accessible values.

Before we start let us note that there are three energy scales, $T, \mu, \omega_{z}$ in the system ( $\mu$ without an argument refers to the chemical potential at the center of the trap, and we are neglecting $\omega_{x}, \omega_{y}$ here). These give rise to two dimensionless ratios, $T / \mu, \omega_{z} / \mu$. Length scales can be obtained from these energy scales using the mass, via the relation, $L=\frac{1}{\sqrt{2 m E}}$.

Thermodynamics in the Trap:

As discussed in Subsection B1 in the presence of a trap the equations for superfluid dynamics can be solved at equilibrium by taking the chemical potential to have a local value which varies along the trap, as given by ${ }^{2}$ Eq. B7. The temperature $T$ in equilibrium is a constant.

Once we have the function $\mathcal{G}$ as discussed in Sec. IIIE, one can then use LDA to express all quantities of interest as a function of the displacement from the trap center (which we denote by r). Thus, within LDA, we can write the number density as

$$
n(\mathbf{r})=n(\mu(\mathbf{r}), T)
$$

We can also express energy and entropy density in the same fashion as a function of the distance from the trap center. Some comments on the conditions for the violation of LDA will be made in the end of the section.

To set the scales we show (see Fig. 6) the number density and the entropy density as a function of the distance $z$ from the trap center at $x=0, y=0$, for a typical trap configuration that we

\footnotetext{
${ }^{2}$ From now on $\mu$ without the argument $\mathbf{r}$ refers to the chemical potential at the center of the trap and $\mu(\mathbf{r})=\mu-\phi(\mathbf{r})$.
} 
consider. In all the examples we consider, we will take $\mathrm{Li}_{6}$ as the fermionic species.

In making Fig. 6, the chemical potential at the center of the trap is chosen to be $10 \mu \mathrm{K}$ which is typical for experiments performed with fermionic cold atoms [22, 74]. The potential is taken to be harmonic (Eq. 6), with the confinement frequency along $z$ direction, $\omega_{z}=2 \pi \times 10^{4} \mathrm{rads} / \mathrm{s}$ which is about 10 times that chosen in Ref. [74]. ${ }^{3}$ Since we are taking $x=y=0, \omega_{x}$ and $\omega_{y}$ do not matter in drawing Fig. 6. However, since we will be exploring anisotropic traps we keep in mind the condition that $\omega_{x}=\omega_{y} \ll \omega_{z}$.

The temperature throughout the trap is taken to be $T=\frac{2 T_{c}}{3}$, where $T_{c}$ is the critical temperature (Eq. 33) associated with the chemical potential $(\mu)$ at the center of the trap defined by

$$
T_{c} \equiv 0.4 \mu
$$

To avoid confusion we note that $T_{c}$ is the temperature at which the superfluid to normal phase transition would have occurred at the center of the trap. In the system under consideration with $T=\frac{2 T_{c}}{3}$, since $T$ at the center of the trap is below the local critical temperature at the center of the trap, the transition actually occurs away from the center of the trap, at a location $z=z_{c}$, where the local chemical potential $\mu\left(z_{c}\right)=\frac{T}{(0.4)}$ [where we have abbreviated $\mu\left(\left(0,0, z_{c}\right)\right)$ as $\mu\left(z_{c}\right)$ ] corresponding to the phase transition to the normal phase. In Fig. 6 we have denoted it by dashed (gray online) vertical lines corresponding to the central value and the error bands.

The error bands to the densities (marked by red curves online surrounding the blue central curve) are associated with the errors in $\mathcal{G}$ (Fig. 4). They are discontinued from $z=27.5 \times 10^{-5}$ $\mathrm{cm}$ corresponding to the point where we switch to Eq. 31 to calculate the thermodynamics.

In the other trap geometries we consider below, we will keep the chemical potential at the center, $\mu$, unchanged as it will set the overall scale of the problem, and only change the temperature of the trap and the confining frequency $\omega_{z}$, in order to explore traps which satisfy criteria listed in Sec. II. The strategy we follow is given below.

As explained in the last section, we estimate the $\eta$ at a given location $\mathbf{r}$ corresponding to the local chemical potential $\mu(\mathbf{r})$ and temperature $T$ by simply multiplying the local number density $n$ we find using Eq. 28 with $\frac{\eta}{n}$ from Fig. 3 of Ref. [23]. (We have reproduced it here in Fig. 5 for convenience.) This estimate assumes that not only thermodynamic but also the transport quantities are determined by the local chemical potential and the temperature. This estimate

\footnotetext{
${ }^{3}$ For conversions to energy units, we use $1 \mathrm{eV}^{-1}=1.97 \times 10^{-7} \mathrm{~m}, 1 \mathrm{eV}=1.78 \times 10^{-36} \mathrm{~kg}, 1 \mathrm{eV}^{-1}=6.58 \times 10^{-16}$ $\mathrm{s}, 1 \mathrm{eV}=1.16 \times 10^{4} \mathrm{~K}$. The mass of $\mathrm{Li}_{6}$ in natural units is $5.6 \times 10^{9} \mathrm{eV}$.
} 

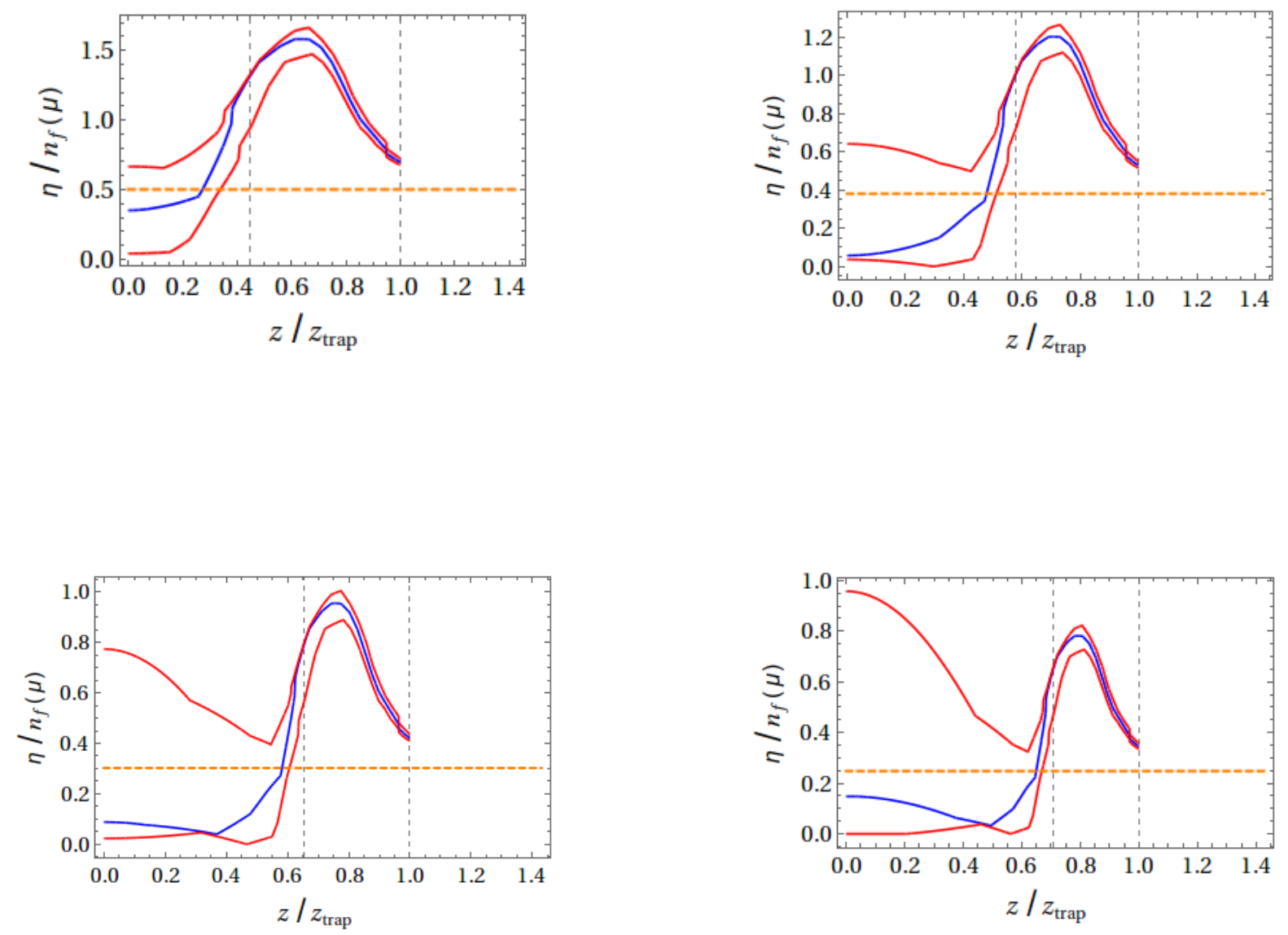

FIG. 7: (Color online) Local shear viscosity with respect to $z$ for $T=\frac{4 T_{c}}{5}$ (top left), $T=\frac{2 T_{c}}{3}$ (top right) $T=\frac{4 T_{c}}{7}$ (bottom left) and $T=\frac{T_{c}}{2}$ (bottom right) at $\omega_{z}=2 \pi \times 10^{4} \mathrm{rads} / \mathrm{s}$ and $\mu=10 \mu \mathrm{K}$. The red curves around the central blue curve denote the error estimate which include errors in the measurement of $\eta / n[23$. as well as errors in $\mathcal{G}$ due to errors in the measurements of thermodynamics 63. The black dashed vertical line to the left is at $z_{c}$ while the one to the right is at $z_{\text {trap }}=\sqrt{\frac{2 \mu}{m \omega_{z}^{2}}}$. We do not extend the viscosity curves in the dilute regime as discussed in Section III C. The dashed orange horizontal line corresponds to $\eta / n_{f}$ in the $\mu \rightarrow-\infty$ limit ([56]).

necessarily implies that the viscosity is isotropic. Nonetheless this will help us identify the values of $T / \mu$ for which the energy loss of the hydrodynamic shear modes is dominated by a region where the potential can be approximated as a linear potential. Having done that, we will increase $\omega_{z}$ to induce anisotropy in the transport coefficients.

Let us consider the four panels in Fig. 7. They show the local shear viscosity (in units of $(2 m \mu)^{3 / 2} /\left(3 \pi^{2}\right)$ where $\mu$ is the central chemical potential) as a function of $z$ for $x=0, y=0$ for four different temperatures at $\omega_{z}=2 \pi \times 10^{4} \mathrm{rads} / \mathrm{s}$. The chemical potential at the center is taken 
to be $10 \mu \mathrm{K}$. The temperatures are $T=\frac{4 T_{c}}{5}$ (top left panel), $T=\frac{2 T_{c}}{3}$ (top right panel) and $T=\frac{4 T_{c}}{7}$ (bottom left panel) and $T=\frac{T_{c}}{2}$ (bottom right panel). Like Fig. 6, the vertical line (gray online) corresponds to $z_{c}$ where $T=0.4 \mu\left(z_{c}\right)$. The error bands of the curves are associated with the errors in $\mathcal{G}$ - which impact $n$ - as well as the errors in the measured $\eta / n$. The $x$-axes of the plots is the $z$ coordinate scaled by the trap size

$$
z_{\text {trap }}=\sqrt{\frac{2 \mu}{m \omega_{z}^{2}}} .
$$

One can also define a characteristic distance $z_{\max }$ where $T / \mu(z)=1$ given by

$$
z_{\max }=\sqrt{\frac{2(\mu-T)}{m \omega_{z}^{2}}} .
$$

For $\mu=10 \mu \mathrm{K}$ at the center of the trap and $\omega_{z}=2 \pi \times 10^{4} \mathrm{rads} / \mathrm{s}, z_{\text {trap }}$ and $z_{\text {max }}$ are $\sim 10^{-4} \mathrm{~cm}$. Beyond the distance $z_{\text {trap }}$, we assume the viscosity to behave like $\frac{15}{32 \sqrt{\pi}}(m T)^{\frac{3}{2}}$ as predicted by the two-body Boltzmann equation [56].

Note that within LDA the plots in Fig. 7 are independent of $\omega_{z}$ if we keep $T / T_{c}$ fixed. This is because scaling $\omega_{z}$ by a factor $f$ can be undone by scaling $z$ by a factor $1 / f$. Since $z_{\text {trap }}$ is scaled by the same factor, $z / z_{\text {trap }}$ at any point on the curve remains unchanged.

To understand the behavior of viscosity along the trap, first consider the central values in Fig. 7 (blue curve online). For all temperatures given above (notice that they are all below $T_{c}$ meaning that the centre of the trap is superfluid), we find the presence of a peak in the middle region of the trap length. Qualitatively we understand this from the fact that the local entropy (see Eq. 28) is the product of $n_{f}(\mu(\mathbf{r}))$ which decreases along the length of the trap, while the function $\mathcal{G}^{\prime}$ increases along the length of the trap, hence it is natural to expect a peak for the entropy density somewhere along the length of the trap. It is clearly seen in the right panel of Fig. 6. Since the local shear viscosity over entropy density is relatively slowly varying in this region (the peak location is just above the critical region), it is not surprising that the local shear viscosity shows a similar behavior. Henceforth, we will denote the position of this peak by $z_{0}$. We also denote the full width at half maximum of the peak by $\delta z$.

The existence of the peak allows us to construct a system where the dominant contribution comes from a region where the potential approximately varies linearly, modeling the theories (Sec. II) where the force that breaks rotational invariance is spatially constant. Here, the trap potential is harmonic, but the dominant contribution to the integral in Eq. 11 and Eq. 12 comes from an 
interval $\delta z$ near $z_{0}$. If we expand the confinement potential as a Taylor series around $z_{0}$ as

$$
\phi\left(z_{0}\right)+\phi^{\prime}\left(z_{0}\right)(\delta z)+\frac{1}{2} \phi^{\prime \prime}\left(z_{0}\right)(\delta z)^{2}+\ldots \ldots
$$

The linearity approximation will hold as long as the confinement potential satisfies

$$
\frac{\phi^{\prime \prime}(z)}{\phi^{\prime}(z)} \delta z \ll 1 \Rightarrow l \equiv \frac{\delta z}{z_{0}} \ll 1
$$

Since we are using a harmonic trap, there are no higher order terms. Our criterion for constant driving force is therefore straightforward. We desire that the dimensionless ratio $l \equiv \frac{\delta z}{z_{0}}$ be less than 1.

There are other motivations to choose the dominant contribution to shear viscosity to arise from such a localized region. We are interested in extracting the value of $\eta / s$, for suitable components of the viscosity tensor, for particular values of $T, \mu$ (in particular, close to the critical temperature $T_{c}$ where $\eta / s$ is known to be close to the KSS bound). Due to the varying trap potential, $\mu(z)$ and therefore the entropy density at equilibrium also vary along the trap. The change resulting in the viscosity due to anisotropy should be bigger than the effect due to the variation of the trap potential on $s$, thereby giving rise to the condition,

$$
\frac{\delta \eta}{\eta}>\frac{\partial s}{\partial z} \frac{\delta z}{s}
$$

As we saw in Sec. II] after Eq. 4 the corrections to the viscosity due to anisotropy go like square of the force that generates the anisotropy. For the system at hand this leads to the expectation

$$
\frac{\delta \eta}{\eta} \sim \frac{(\nabla \phi)^{2}}{\left(\mu(z)^{2} k_{F}(z)^{2}\right)} .
$$

This estimate agrees with the analysis based on the Boltzmann equation as discussed later in Sec.IV (see Eq. 65). The RHS in Eq. 40 goes like $\frac{\partial s}{\partial z} \frac{\delta z}{s} \sim \delta z / z_{0}=l$, and this gives rise to the condition

$$
\kappa_{\mathrm{LDA}}^{2}>l
$$

where we have introduced the notation

$$
\kappa_{\mathrm{LDA}}=\frac{(\nabla \phi)}{\left(\mu\left(z_{0}\right) k_{F}\left(z_{0}\right)\right)}
$$


It is easy to see that $\kappa_{\mathrm{LDA}}$ roughly scales as

$$
\kappa_{\mathrm{LDA}} \sim \frac{\omega_{z}}{\mu}
$$

so that Eq. 42 leads to the condition

$$
\frac{\omega_{z}^{2}}{\mu^{2}}>l
$$

For fixed $T, \mu$ one can show that $l$ does not change as $\omega_{z}$ changes. Thus the left hand side is independent of the ratio $\frac{\omega_{z}}{\mu}$ for fixed $T / \mu$, and the inequality can be met for sufficiently large $\frac{\omega_{z}}{\mu}$.

Let us also mention that the gravity results apply to situations with only linearly varying potential (Eq. 2) leading to only $|\nabla \phi|^{2}$ corrections due to the anisotropy. In general we would expect that there are additional corrections proportional to $\nabla^{2} \phi$. There is little guidance on what these corrections do, for the kind of strongly coupled system we are dealing with here. Thus, to the extent we are trying to stay close to situations where gravitational systems give at least some guidance, it is desirable to choose the dominant contribution to shear viscosity to arise from a narrow localized region.

Viscosity and Other Properties For Varying Trap Parameters: Table II

We now turn to examining the behavior of $\eta, \eta / s$, and $l=\frac{\delta z}{z_{0}}$ as trap parameters are varied. In Table II we keep $\omega, \mu$ fixed to take the values $\omega_{z}=2 \pi \times 10^{4} \mathrm{rads} / \mathrm{s}, \mu=10 \mu \mathrm{K}$ and vary $T$. As mentioned at the beginning of Subsection IIIF there are two dimensionless ratios that characterize the energy scales in this system. The different rows corresponding to different values of $T$ in units of $T_{c}$ show how various quantities vary with $T / \mu$. The scaling of these quantities with $\omega_{z} / \mu$ is given in the first line on top of the Table. II. Thus $\kappa_{\text {LDA }}$ scales like $\omega_{z} / \mu . z_{0}, z_{\text {trap }}$ and $\delta z$ scale like $1 / \omega_{z}$ for fixed $T, \mu$, as was discussed above after Eq. 37. Thus their ratios, $\frac{z_{o}}{z_{\text {trap }}}, l=\frac{\delta z}{z_{0}}$ etc. are independent of $\omega_{z} / \mu$. The third column of the Table. II tests the linearity of the potential, which is a good approximation near the peak if $l=\delta z / z_{0} \ll 1$.

The ratio $l$ is governed by the temperature of the trap divided by the chemical potential or equivalently $T_{c}$ at the center. As we decrease $T / T_{c}, z_{0}$ increases and $\delta z$ decreases. This consideration would suggest that to obtain $\frac{\delta z}{z_{0}}$ as small as possible we should consider as small a temperature as possible. But this conclusion is not correct as is clear from the upper error band in Fig. 7) (red online).

The errors bands on $\eta$ are fairly narrow in the region near $z_{0}$. However, the errors grow near 


\begin{tabular}{|c|c|c|c|c|c|c|c|}
\hline$T$ & $z_{\operatorname{trap}} \sqrt{\frac{\mu}{10 \mu \mathrm{K}}} \frac{2 \pi \times 10^{4}}{\omega} \mathrm{cm}$ & $\frac{z_{0}}{z_{\text {trap }}}$ & $l$ & $\left.\frac{T}{\mu(z)}\right|_{z_{0}}$ & $\left.\frac{\eta}{n}\right|_{z_{0}}$ & $\left.\frac{\eta}{s}\right|_{z_{0}}$ & $\kappa_{\mathrm{LDA}} \frac{10 \mu \mathrm{K}}{\mu} \frac{\omega_{z}}{2 \pi \times 10^{4} \mathrm{rad} / \mathrm{s}}$ \\
\hline $4 T_{c} / 5$ & $27 \times 10^{-5}$ & 0.63 & 0.98 & 0.54 & 0.89 & 0.85 & 0.05 \\
$2 T_{c} / 3$ & $27 \times 10^{-5}$ & 0.71 & 0.62 & 0.54 & 0.89 & 0.85 & 0.08 \\
$4 T_{c} / 7$ & $27 \times 10^{-5}$ & 0.76 & 0.46 & 0.54 & 0.89 & 0.85 & 0.11 \\
$T_{c} / 2$ & $27 \times 10^{-5}$ & 0.8 & 0.37 & 0.55 & 0.91 & 0.85 & 0.13 \\
\hline
\end{tabular}

TABLE II: Trap characteristics for various $T / T_{c}$. The scaling behavior of various quantities with $\omega_{z}$ are also shown. The entries were calculated for $\mu=10 \mu \mathrm{K}, T_{c}=0.4 \mu . l=\frac{\delta z}{z_{0}}$ (Eq. 39 tests how well the potential can be approximated as a linear potential in the regime of interest. $\kappa_{\text {LDA }}$ (Eq. 59 ) tests how well LDA is expected to work at $z_{0}$.

\begin{tabular}{|c|c|c|c|c|c|c|c|}
\hline$T$ & $\alpha_{x}^{\max }\left(10^{-10} \mathrm{eV}\right)$ & $\dot{E}_{\text {kinetic }}(\mathrm{j} / \mathrm{s})(\mathbf{a})$ & $E(\mathrm{j})(\mathbf{a})$ & $\tau_{0}(s)(\mathbf{a})$ & $\dot{E}_{\text {kinetic }}(\mathrm{j} / \mathrm{s})(\mathbf{b})$ & $E(\mathrm{j})(\mathbf{b})$ & $\tau_{0}(s)(\mathbf{b})$ \\
\hline $4 T_{c} / 5$ & 2.83 & $2.37 \times 10^{-16}$ & $3 \times 10^{-20}$ & 0.0002 & $4.7 \times 10^{-16}$ & $10^{-17}$ & 0.04 \\
$2 T_{c} / 3$ & 2.35 & $1.25 \times 10^{-16}$ & $2 \times 10^{-20}$ & 0.0003 & $2.5 \times 10^{-16}$ & $6.8 \times 10^{-18}$ & 0.05 \\
$4 T_{c} / 7$ & 2.02 & $7.12 \times 10^{-17}$ & $1.4 \times 10^{-20}$ & 0.0004 & $1.4 \times 10^{-16}$ & $4.8 \times 10^{-18}$ & 0.07 \\
$T_{c} / 2$ & 1.77 & $4.33 \times 10^{-17}$ & $1.1 \times 10^{-20}$ & 0.0005 & $8.65 \times 10^{-17}$ & $3.6 \times 10^{-18}$ & 0.08 \\
\hline
\end{tabular}

TABLE III: Additional trap characteristics for various $T / T_{c}$ at $\omega_{z}=2 \pi \times 10^{4}$ rads $/ \mathrm{s}, \omega_{x}=\omega_{y}=2 \pi \times 385$ rads/s and $\mu=10 \mu \mathrm{K}$. The energy is given in joules abbreviated as ' $\mathrm{j}$ ' and energy loss rate in joules per second, $(\mathrm{j} / \mathrm{s})$. For a fixed $T / \mu$, the energy of the Elliptic mode scales as $\sim \frac{1}{\omega_{x} \omega_{y} \omega_{z}^{3}}$ and that of the Scissor mode scales as $\sim \frac{1}{\omega_{x}^{3} \omega_{y} \omega_{z}}$. The characteristic time $\tau_{0}$ ( given in seconds 's' in the table and defined in Eq 50 , of the Elliptic mode scales as $\sim \frac{\mu}{\omega_{z}^{2}}$ and that of the Scissor mode scales as $\sim \frac{\mu}{\omega_{x}^{2}}$. For the Elliptic mode to account for the fact that only the normal component of the velocity is non-zero near the trap centre, we assume that the normal component density in this region is $\frac{T}{T_{c}}$ times the total density in this region. For the Scissor mode we have the full number density.

$z \rightarrow 0$, in particular for smaller $T / T_{c}$ (Fig. 7). The reason is the large errors in the measured $\eta / n$ in the superfluid regime (see the region $T / T_{F} \lesssim 0.16$ in Fig. 5). Indeed, we expect that for $T \ll T_{F}$, the viscosity is dominated by superfluid phonons whose contribution diverges as $T \rightarrow 0$ as $\eta \approx\left(9.3 \times 10^{-6}\right) \xi^{5}\left(T_{F}^{8} / v^{3} T^{5}\right)$ where $v$ is the speed of superfluid phonons 64. Numerically, $\eta / n \approx 2.5 \times 10^{-5} \frac{T_{F}^{5}}{T^{5}}$. Therefore, to avoid a large contribution from the center of the trap rather than from near $z_{0}$, we do not consider temperatures below $T_{c} / 2$. Within this constrained temperature regime between $T_{c} / 2$ and $T_{c}$ we find that the linearity condition $\delta z / z_{0}<1$ is satisfied, although it is not possible to generate traps where $\delta z / z_{0}$ is parametrically small. In the narrow range of temperatures, it turns out that the location of $z_{0}$ is such that $T / \mu\left(z_{0}\right) \approx 0.54$, just off to the right of the phase transition at $T / \mu\left(z_{c}\right) \approx 0.4$.

Note that, as explained in the discussion above, a few paragraphs after Eq. 35, the value for the viscosity $\eta / s$ which appears in the Table II is an approximate one, obtained by taking the value in the isotropic situation corresponding to the local value for $\mu, T$ at the location $z_{0}$. By a similar argument as before, this value is independent of the ratio $\omega_{z} / \mu$ for a fixed $T / T_{c}$. We note that the 
values of $\eta / s$ in the Table $\mathrm{II}$ are about 10 times the KSS bound. One would expect that various components of the viscosity tensor deviate from this rough value by a fraction of order $\kappa_{\mathrm{LDA}}^{2}$. The parameter $\kappa_{\text {LDA }}$ which was introduced in Eq. 43 above, when computed at the location of the peak $z_{0}$, has the more exact form

$$
\kappa_{\mathrm{LDA}}=\frac{m \omega_{z}^{2} z_{0}}{\left(3 \pi^{2} n\left(z_{0}\right)\right)^{\frac{1}{3}} \mu\left(z_{0}\right)}=\frac{\sqrt{\frac{m}{2}} \omega_{z}^{2} z_{0}}{\left[\mathcal{F}\left(\frac{T}{\mu\left(z_{0}\right)}\right)\right]^{1 / 3}\left[\mu\left(z_{0}\right)\right]^{\frac{3}{2}}}
$$

as one can easily check by using Eq. 28 ,

\section{Energy Damping For Varying Values of Trap Parameters: Table III}

We now turn to considering the effects of varying the trap parameters on various quantities like the total energy $E_{\text {kinetic }}$, the damping rate of this energy $\dot{E}_{\text {kinetic }}$, etc. In Table III we again keep $\mu, \omega_{z}$ fixed to take values $\omega_{z}=2 \pi \times 10^{4} \mathrm{rads} / \mathrm{s}, \mu=10 \mu \mathrm{K}$ and consider the effects of varying $T$. In addition, we also need to consider the effects of the harmonic trap in the $x, y$ directions. We keep $\omega_{x}, \omega_{y}$ to be fixed to take values $\omega_{x}=\omega_{y}=2 \pi \times 385 \mathrm{rads} / \mathrm{s}$. The different rows then give how various quantities vary as $T / \mu$ changes. We note that for the range of temperatures considered the total number of atoms in the trap is approximately, $\sim 10^{6}$.

The energy which appears in this Table is the total mechanical energy $E$ given by

$$
E=2 E_{\text {kinetic }}
$$

where

$$
E_{\text {kinetic }}=\left\langle\frac{1}{2} \int d^{3} \mathbf{r} m n(\mathbf{r}) \mathbf{v}^{2}\right\rangle
$$

where $\mathbf{v}$ is the velocity of either mode and the average is taken over one cycle for the scissor mode (the elliptic mode is non-oscillatory). For the Elliptic mode and the Scissor mode with amplitude $\alpha_{x}^{\max }$, the kinetic energy is given as follows:

$$
\begin{aligned}
& \text { For Elliptic, } E_{\text {kinetic }}(\mathbf{a})=\int d^{3} \mathbf{r} \frac{1}{2} m n_{\text {normal }}\left(\alpha_{x}^{\max }\right)^{2}\left[\frac{\omega_{x}^{4}}{\omega_{z}^{4}} x^{2}+z^{2}\right] \\
& \text { For Scissor, } E_{\text {kinetic }}(\mathbf{b})=\int d^{3} \mathbf{r} \frac{1}{4} m n\left(\alpha_{x}^{\max }\right)^{2}\left[x^{2}+z^{2}\right] .
\end{aligned}
$$

$\dot{E}_{\text {kinetic }}$ is the rate of energy loss due to viscosity induced dissipation, Eq. 10. The energy loss, $\dot{E}_{\text {kinetic }}$ in these modes is given by Eqns. 11,12 . 
Note that for the Scissor mode the expression corresponds to the kinetic energy averaged over an oscillation cycle. Also, for the Elliptic mode, $v_{s}=0$, Eq. 8, and only the normal component contributes to the kinetic energy. The density in the normal phase is estimated in the region close to the centre, where both the superfluid and normal components are present, as being $\frac{T}{T_{c}}$ times the total density in this region and we have denoted it by $n_{\text {normal }}$ in Eq. 49 . For the Scissor mode we have the full number density denoted by $n$ in the above formulas.

The validity of hydrodynamics imposes a condition on how big $\alpha_{x}$ can become, the resulting maximum value, $\alpha_{x}^{\max }$ was estimated in Eq. 16 . The quantities $E_{\text {kinetic }}, \dot{E}_{\text {kinetic }}$ which appear in Table III are obtained from Eq. 10, Eq. 49 by setting $\alpha_{x}=\alpha_{x}^{\max }$.

A convenient quantity with which to compare $\alpha_{x}^{\max }$ is the ratio of the speed of sound at the centre $c_{s}=\sqrt{\frac{2 \mu}{3 m}}$ to a measure of the trap size $z_{\text {trap }}$. For comparison, let us note that for $\omega_{z}=2 \pi \times 10^{4}$ rads/s we obtain $\frac{c_{s}}{z_{\text {trap }}}=\frac{\omega_{z}}{\sqrt{3}}=3.63 \times 10^{-11} \mathrm{eV}$.

The (amplitude) damping time $\tau_{0}$, which appears in Table III, is defined as

$$
\tau_{0}=2 E / \dot{E}_{\text {kinetic }}
$$

As mentioned above, the table considers the effects of varying the temperature while keeping $\mu, \omega_{z}, \omega_{x}, \omega_{y}$ fixed. For fixed $T / \mu$ one can also consider what happens as the angular frequencies are varied. In the highly anisotropic situations $\omega_{z} \gg \omega_{x}, \omega_{y}$, one finds that the total energy $E_{\text {kinetic }}$ for the Elliptic mode approximately scales like

$$
E_{\text {kinetic }}(a) \sim \mu \frac{\mu}{\omega_{x}} \frac{\mu}{\omega_{y}}\left(\frac{\mu}{\omega_{z}}\right)^{3}
$$

and the damping time $\tau_{0}$ for the Elliptic mode approximately scales like

$$
\tau_{0}(a) \sim \frac{\mu}{\omega_{z}^{2}}
$$

Similarly for the Scissor mode we get

$$
E_{\text {kinetic }}(b) \sim \mu \frac{\mu}{\omega_{y}} \frac{\mu}{\omega_{z}}\left(\frac{\mu}{\omega_{x}}\right)^{3}
$$




$$
\tau_{0}(b) \sim \frac{\mu}{\omega_{x}^{2}}
$$

These scalings are obtained by noting that $\alpha_{x}^{\max } \sim \mu$ for fixed $T / \mu$, and also that the trap potential is unchanged under a rescaling $\omega_{z} \rightarrow \lambda \omega_{z}, z \rightarrow z / \lambda$ and similarly for $x, y$. We have also assumed that $\omega_{z} \gg \omega_{x}, \omega_{y}$. Some of these scalings are summarized in the caption below Table III. For example, the scalings of the scissor mode, can be derived as follows: $E \sim \int d x d y d z\left[m n v^{2}\right] \sim$ $L_{x} L_{y} L_{z}\left[m n \alpha^{2} L_{x}^{2}\right] \sim \frac{\mu^{6}}{\omega_{x}^{3} \omega_{y} \omega_{z}}$, where we have assumed that at the center of the trap $\mu>0$ and $L_{i}=\sqrt{2 \mu /\left(m \omega_{i}^{2}\right)}$.) In a similar manner, one can derive the approximate scalings for energy dissipation rates: $\dot{E} \sim \frac{\mu^{5}}{\omega_{x} \omega_{y} \omega_{z}}$ for both the modes (assuming $\eta$ scales the same way as $n$ ie. $\sim(m \mu)^{\frac{3}{2}}$.

The approximate value of $T, \mu, \omega_{z}$ we consider here are of the same order as those considered in 22] where the viscosity of a unitary Fermi gas was measured, using a radial breathing mode. The Scissor mode has been considered in the literature before. The damping rate has been measured for cold atoms system in this mode in superfluid bosonic (see Ref. [48] and Refs. therein) and in fermionic systems [49]. In particular [49] carries out these measurements in the unitary Fermi gas. The values for trap parameters we consider are similar to those considered for example in [22] and not very different from those considered in [49]. The maximum angular amplitude of the the scissor mode is determined by the velocity amplitude $\alpha_{x}$ (Eqs. 9, 7) which is bounded above by $\alpha_{x}^{\max }$ in Table III. One can show that the angular amplitude (in radians) of the oscillation executed by the deformed cloud in the scissor mode is given by

$$
\theta=\tan ^{-1}\left(\frac{e^{\frac{2 \alpha_{x}}{\omega}}-1}{e^{\frac{2 \alpha_{x}}{\omega}}+1}\right)
$$

where $\omega=\sqrt{\omega_{x}^{2}+\omega_{z}^{2}}$. Taking $\alpha_{x}$ to be the maximum value $\alpha_{x}^{\max } \sim 10^{-10} \mathrm{eV}$ and $\omega$ to be $2 \pi \times 10^{4}$ rads/s $\equiv 4.16 \times 10^{-11} \mathrm{eV}$, we find $\theta_{\max } \sim \tan ^{-1}[1] \equiv 45^{\circ}$. For a frequency 10 times larger, $\theta_{\max } \sim \tan ^{-1}[0.4] \equiv 24^{\circ}$. It is satisfying that these amplitudes are larger than those measured in [49] for the scissor mode and hence the condition for hydrodynamics (Eq. 16) does not force the amplitudes to be so small as to preclude observation using existing techniques. For $\mu=10 \mu K$, $\omega_{x}=\omega_{y}=2 \pi \times 385 \mathrm{rads} / \mathrm{s}$ and $\omega_{z}=2 \pi \times 10^{4} \mathrm{rads} / \mathrm{s}, \tau_{0}$ ranges from roughly 0.04 sec to 0.08 sec. The damping of the scissor mode has been observed for slightly different parameters values, $\mu \approx 1 \mu K, \omega_{x}=2 \pi \times 830 \mathrm{~Hz}, \omega_{y}=2 \pi \times 415 \mathrm{~Hz}$ and $\omega_{z}=2 \pi \times 22 \mathrm{~Hz}$ in Ref. [49] where the 
damping time scales measured are of the order of milliseconds.

\section{Summary:}

Now we come to the punch line of this section. The effects of anisotropy can cause a fractional change in components of the viscosity tensor, potentially lowering some of them. This effect is expected to go like, $\delta \eta / \eta \sim \kappa_{\mathrm{LDA}}^{2}$, as mentioned in Eq. 41. We see from Table II that, for fixed $\omega_{z} / \mu, \kappa_{\mathrm{LDA}}$ increases as $T$ decreases (i.e. $T / \mu$ decreases), with the maximum value, within the range of allowed temperatures, being of order $\kappa_{\mathrm{LDA}} \sim 10 \%$. This would lead, one expects, to a fractional change in components of the viscosity of order $\delta \eta / \eta \sim($ few $) \times 1 \%$, which is quite small. However note that increasing $\omega_{z}$ will increase $\kappa_{\mathrm{LDA}}$ with a linear dependence $\kappa_{\mathrm{LDA}} \sim \omega_{z} / \mu$ as noted in Eq. 44 and also in the first row of Table II. In turn this should lead to a quadratic fractional change in $\delta \eta / \eta \sim\left(\frac{\omega_{z}}{\mu}\right)^{2}$. We can carry out this change while keeping $\omega_{x}, \omega_{y}$ fixed thereby increasing the anisotropy. Note that this change of $\omega_{z}$ will decrease the total energy of this mode $E_{\text {kinetic }}(b) \sim 1 / \omega_{z}$, Eq 53 , but it does not change $\tau_{0}$ significantly, since $\tau_{0}$ depends to a good approximation on $\omega_{x}$ and not $\omega_{z}$ as seen from Eq. 54. Also note that changing $\omega_{z}$ while keeping $T / \mu$ fixed will not change $l$ and thus the localized nature of the region from which the damping arise. In fact it will make it easier to meet the condition Eq. 45 .

Also it is worth commenting that it is easy to see from Eq. 44 Eq. 53 and Eq. 54 that if one want to keep $\tau_{0}$ and $E_{\text {kinetic }}$ for the scissor mode both fixed and increase $\kappa_{\text {LDA }} \rightarrow \lambda \kappa_{\text {LDA }}$ one could do this (while keeping $\omega_{x}=\omega_{y}$ ) by scaling

$$
\omega_{x} \rightarrow \lambda^{\frac{1}{6}} \omega_{x}, \omega_{y} \rightarrow \lambda^{\frac{1}{6}} \omega_{y}, \omega_{z} \rightarrow \lambda^{\frac{4}{3}} \omega_{z}, \mu \rightarrow \lambda^{\frac{1}{3}} \mu, T \rightarrow \lambda^{\frac{1}{3}} T .
$$

This keeps $\frac{T}{\mu}, \tau_{0}$ and $E_{\text {kinetic }}$ fixed, increases the overall magnitude of $\mu$, increases $\omega_{z}$ and also $\omega_{x}, \omega_{y}$

The discussion of the previous two paragraphs suggests that one can quite plausibly keep the damping time scale and the total energy in the experimentally accessible range, while gradually increasing $\omega_{z}$ making $\kappa_{\mathrm{LDA}} \sim \mathcal{O}(1)$ and the effects of anisotropy significant. While some of the theoretical approximations made will break down in this limit it is possible that the effects of anisotropy would get more pronounced, and potentially even dramatic, driving the spin one components of the viscosity to be much smaller than their values in the isotropic case, and potentially even violating the KSS bound.

We have not discussed the Elliptic mode in as much detail. One reason is that unlike the scissor 
mode, this mode has not been experimentally realized in cold atom systems yet. ${ }^{4}$ Also we see from Table III that the damping time $\tau_{0}$ in this case is about two orders of magnitude smaller, and this too might be an issue of some experimental concern. It may of course turn out that this mode is experimentally accessible. It will then be certainly interesting to explore its properties, especially since this mode in a very direct way measures the resistance to shear in the resulting fluid flow.

Finally we note that all the five conditions which were listed at the end of Sec. II for observing the suppression of viscosity can be met in the system being analyzed here. Conditions 1 and 2 are met by the two modes discussed above in the unitary Fermi gas. We have ensured that $l<1$ (Table II) so that the contribution arises from a localized region where the potential is approximately linear, meeting condition 4. As argued above, for the scissors mode the anisotropy can be made large enough while staying within the fluid mechanics approximation $\left(\alpha_{x}<\alpha_{x}^{\max }\right)$ thereby meeting conditions 3 and 5 . The resulting values for the total energy and the damping time we find lie within the experimentally accessible range.

To summarize, we have seen in this section that for experimentally reasonable values of parameters one can increase the anisotropy of the trapping potential and probe the viscosity tensor by measuring the energy loss and related damping time in the scissor mode. As the anisotropy is increased, its effects could well become quite significant driving some components of the viscosity (spin 1 in our notation) to become very small, and potentially making them even smaller than the KSS bound.

\section{G. Discussion on $\kappa_{\text {LDA }}$}

In this subsection, we present a detailed discussion on $\kappa_{\text {LDA }}$ given in the last column of Table. III. The results discussed so far assume LDA is valid. LDA rests on the assumption that the trap potential varies slowly on the scale of the local Fermi wavelength $k_{F}^{-1}(\mathbf{r})=\left(3 \pi^{2} n(\mathbf{r})\right)^{\frac{1}{3}}$ ie. at any local point $\mathbf{r}$ along the length of the trap, the following condition holds true -

$$
\left|\nabla_{\mathbf{r}}(\mu(\mathbf{r})) \frac{1}{k_{F}(\mathbf{r})}\right|_{\mathbf{r}} \ll \mu(\mathbf{r})
$$

\footnotetext{
${ }^{4}$ One possible way to set up the elliptic mode is to start with a more circular trap and exciting a rotational mode by using rotating lasers using a set up similar to Ref. [75. If the rotational frequency is small enough, vortices will not be excited and only the normal fluid will rotate like a rigid body. On adiabatically deforming the trap one would then get the elliptic mode because during adiabatic deformations, hydrodynamics is satisfied at each time and we expect that the normal fluid will go smoothly from circular rotation to the elliptic mode.
} 
Since we desire $\omega_{x}, \omega_{y} \ll \omega_{z}$, the gradient is strongest in the $z$ direction and hence taking $x, y=0$ and moving along the harmonic trap in the $z$ direction, $\frac{d(\mu(z))}{d z}=-m \omega_{z}^{2} z$, we note that LDA violations will be significant if

$$
m \omega_{z}^{2} z \frac{1}{\left(3 \pi^{2} n(z)\right)^{\frac{1}{3}}} \sim \mu(z) .
$$

For any trap geometry at the outer edges of the trap when the density becomes small enough, LDA will be violated $\left(\mu(z)<0\right.$ for $\left.z>z_{\text {trap }}\right)$. These regions typically do not contribute significantly to the trap energy loss. But focusing on the region near $z_{0}$, LDA is a good approximation if

$$
\kappa_{\mathrm{LDA}}=\frac{\sqrt{\frac{m}{2}} \omega_{z}^{2} z_{0}}{\left[\mathcal{F}\left(\frac{T}{\mu\left(z_{0}\right)}\right)\right]^{1 / 3}\left[\mu\left(z_{0}\right)\right]^{\frac{3}{2}}} \ll 1
$$

Approximating $\left.\mathcal{F}\left(\frac{T}{\mu\left(z_{0}\right)}\right)\right]^{1 / 3} \approx \frac{1}{\sqrt{\xi}}$ [Since $\mathcal{F}(0)=1 / \xi^{3 / 2}$, and the deviations from $\mathcal{F}(0)$ are small for $T / \mu \lesssim 1]$, we find

$$
\kappa_{\mathrm{LDA}}=\frac{\sqrt{\frac{m}{2}} \omega_{z}^{2} z_{0}}{\left[\mu\left(z_{0}\right)\right]^{\frac{3}{2}}} \sqrt{\xi} \ll 1,
$$

Since $z_{0}$ scales as $1 / \omega_{z}$ for fixed $\mu$ and $T$, LDA will be violated at $z_{0}$ if $\omega_{z}$ is large enough. From Table II one can see that for $\mu=10 \mu \mathrm{K}$ and $T=T_{c} / 2, \kappa_{\mathrm{LDA}}>1$ for $\omega_{z}>2 \pi \times 77000 \mathrm{rads} / \mathrm{s}$. Alternatively, taking $\omega_{z}=2 \pi \times 10^{4} \mathrm{rads} / \mathrm{s}$ and $T=T_{c} / 2, \kappa_{\mathrm{LDA}}$ can become larger than 1 if $\mu<1.3 \mu \mathrm{K}$.

For $T \rightarrow 0$ the corrections to LDA have been previously studied in Refs. [76, 77]. One can write

$$
n(\mathbf{r})=n_{\mathrm{LDA}}\left(1-\frac{c_{\chi}}{64} \frac{(\nabla \phi(\mathbf{r}))^{2}+4(\mu-\phi(\mathbf{r})) \nabla^{2} \phi(\mathbf{r})}{m(\mu-\phi(\mathbf{r}))^{3}}+\mathcal{O}\left(\nabla^{3} \phi(\mathbf{r})\right)\right)
$$

where $c_{\chi}$ is related to the response of the density to a periodic fluctuation in the potential. The low energy constant $c_{\chi}$ has not been calculated using ab-initio techniques so far. In all model calculations $c_{\chi} \sim 1$, including in a sophisticated analysis using SLDA (Ref. [77]).

For finite $T$ for an isothermal system, the deviations from LDA are not related to the density response but for $T \lesssim(\mu-\phi(\mathbf{r}))$ we can write corrections to LDA in analogy with Eq. 60

$$
n(\mathbf{r})=n_{\mathrm{LDA}}\left(1-\frac{c_{1}}{64} \frac{(\nabla \phi(\mathbf{r}))^{2}}{m(\mu-\phi(\mathbf{r}))^{3}}-\frac{c_{2}}{16} \frac{\nabla^{2} \phi(\mathbf{r})}{m(\mu-\phi(\mathbf{r}))^{2}}+\mathcal{O}\left((\nabla V)^{3}\right)\right)
$$


where $c_{1,2}$ are functions of $(T / \mu)$ and tend to 1 as $T / \mu \rightarrow 0$. In particular, for the interesting region the term proportional to $c_{1}$ is dominant (the exception is near the center of the trap). Therefore, the corrections to LDA near $z_{0}$ can be written as

$$
n(z)=n_{\mathrm{LDA}}\left(1-\frac{c_{1}}{64} \frac{2}{\xi} \kappa_{\mathrm{LDA}}^{2}+\cdot \cdot\right)
$$

where we have used the low temperature expression

$$
m \mu(\mathbf{r})=\frac{\xi}{2} k_{F}^{2}(\mathbf{r})
$$

to write the correction in terms of $\kappa_{\mathrm{LDA}}$.

In the absence of further information about $c_{1}$ at finite $T$ it is difficult to make precise statements about the relevance of LDA corrections for the traps with large values of $\omega_{z}$ that we show in the next Section are needed to make the shear viscosity tensor locally anisotropic. Therefore, we simply use $\kappa_{\mathrm{LDA}} \gtrsim 1$ as a marker for significant LDA violation. However, it is important to keep in mind that if $c_{1}\left(\frac{T}{\mu\left(z_{0}\right)}\right) \sim c_{1}(0.54) \sim 1$ ( ince $\frac{T}{\mu\left(z_{0}\right)} \sim 0.54$ for the cases we consider), then the pre-factor of $1 /(32 \xi)$ implies that the corrections to LDA can be small even for $\kappa_{\text {LDA }} \approx 1$.

\section{LOCAL ANISOTROPY}

Hydrodynamics is an effective theory: The conserved currents are written as a series of terms ordered by the number of derivatives acting on the local fluid velocity. The lowest order terms are simply given by the Galilean (for non-relativistic systems) or Lorentz (for relativistic systems) transforms of the local thermodynamic properties like the density and the pressure, from the local rest frame of the fluid to the laboratory frame. The first order terms are given by the local gradients of the velocity $\left(\partial_{i} u_{j}+\partial_{j} u_{i}\right) / 2$ multiplied by proportionality constants given by the transport coefficients - for example viscosities — of the system. We will not consider higher derivative terms in this paper, instead restricting ourselves to situations (see Eq. 15) where the first order correction is smaller than the lowest order terms.

In the presence of external fields, the law of conservation of energy features a source term proportional to the driving force, $\nabla \phi(\mathbf{r})$. If $\nabla \phi(\mathbf{r})$ is "small" (which we shall define in a moment), its effect on the thermodynamics and transport can be neglected, and hydrodynamics describes a locally isotropic fluid (with isotropic thermodynamic functions and isotropic transport coeffi- 
cients) ${ }^{5}$ moving in a space dependent potential. The key realization therefore is that to observe an anisotropy in thermal or transport properties it is not sufficient for $\omega_{x}, \omega_{y} \ll \omega_{z}$. Corrections to isotropy will start becoming significant as we increase $\omega_{z}$, if $\omega_{z}$ starts becoming comparable to some microscopic scale of the system.

The criterion for the thermodynamic quantities to exhibit the effect of $\nabla \phi(\mathbf{r})$ is clear from the previous section. If the potential varies on length scales comparable to the inter-particle separation - the Thomas-Fermi approximation, or LDA breaks down - the pressure of the fluid in the direction of the gradient will be different from the pressure in the perpendicular directions. In this case, clearly the transport coefficients will also be anisotropic. To explore an analogous system to the one described in Sec. II] this argument prompts us to consider $\omega_{z}$ large enough that LDA is broken (see Table II). For such systems, the estimates for the density Fig. 6 and viscosities Fig. 7 using LDA will be only rough guiding values, but if the analogy with the system in Sec. II] holds true, the viscosity values relevant for the modes described in Sec. III A will be lower than the LDA values, and could be lower than $1 /(4 \pi)$ in suitable quantum units.

To estimate the order of the correction to the shear viscosity due to potential gradients we note that the first order correction to transport due to $\nabla \phi(\mathbf{r})$ simply appear as the source term, and hence assuming that the next order corrections will be analytic in $\nabla \phi(\mathbf{r})$, we expect

$$
\eta_{i j k l}=\eta \frac{1}{2}\left[\left(\delta_{i k} \delta_{j l}+\delta_{i l} \delta_{j k}-\frac{2}{3} \delta_{i j} \delta_{k l}\right)+\left(\frac{\lambda^{2}(\nabla \phi(\mathbf{r}))(\nabla \phi(\mathbf{r}))}{[\mu(\mathbf{r})]^{2}}\right) \sum_{\alpha=0}^{4} c_{(\alpha)} M_{\alpha i j k l}\right]+\mathcal{O}\left(\nabla^{2} \phi,(\nabla \phi)^{4}\right)
$$

where $\lambda$ is a microscopic length scale of the system, $c_{(\alpha)}$ are dimensional constants of order 1 which depend on the microscopic details of the system, and $M_{i}$ are 5 orthonormal projection operators that arise in a system with one special direction (for eg. see Ref. [78]). We have given these projection operators in Appendix. D(Eq. D18).

$\lambda$ is a length scale that determines transport behavior. In a system admitting a quasi-particle description we expect $\lambda$ to be of the order of the mean free path. (We show this explicitly in Appendix. D.) The other length scale in the system is the inter-particle separation $1 / k_{F}$. In terms

\footnotetext{
${ }^{5}$ This assumes that microscopically the fluid is isotropic. For example it is not a crystal [53] or a fluid phase with an anisotropic order parameter.
} 
of $k_{F}$ we can write the corrections as

$$
\begin{aligned}
\eta_{i j k l} & \approx \eta \frac{1}{2}\left[\left(\delta_{i k} \delta_{j l}+\delta_{i l} \delta_{j k}-\frac{2}{3} \delta_{i j} \delta_{k l}\right)+\left(\lambda k_{F}\right)^{2}\left(\frac{(\nabla \phi(\mathbf{r}))(\nabla \phi(\mathbf{r}))}{k_{F}^{2}[\mu(\mathbf{r})]^{2}}\right) \sum_{\alpha=0}^{4} c_{(\alpha)} M_{\alpha i j k l}\right] \\
& =\eta \frac{1}{2}\left[\left(\delta_{i k} \delta_{j l}+\delta_{i l} \delta_{j k}-\frac{2}{3} \delta_{i j} \delta_{k l}\right)+\left(\lambda k_{F}\right)^{2}\left(\kappa_{\mathrm{LDA}}^{2}\right) \sum_{\alpha=0}^{4} c_{(\alpha)} M_{\alpha i j k l}\right]
\end{aligned}
$$

For weakly interacting quasi-particles, the $\lambda k_{F} \gg 1$. But for a strongly interacting system in the absence of more information about $\lambda k_{F}$ and $c_{(\alpha)}$ it is not possible to make a more concrete statement about the corrections to viscosity. We can only state that the corrections are important if $\kappa_{\mathrm{LDA}} \sim 1$ as we did in Eq. 41 .

As discussed in Sec.II, for the theories considered in Sec. III, there is no quasi-particle description. The only relevant length scale is $1 / T$ and the field $\phi$ changes by order 1 on a length scale $1 / \rho$. Using AdS/CFT it has been shown [45] that the corrections to isotropy go as Eq. 4.

For the unitary Fermi gas there is no known gravitational dual [79] and we will need to resort to a rough calculation to estimate $c_{(\alpha)}$ and $\lambda k_{F}$. We solve the Boltzmann transport equation in the relaxation time approximation. We hope this will give semi-quantitative results. We leave the challenging calculation of the viscosity for temperatures in the strongly coupled regime just above the critical temperature in the presence of a background potential for future work.

As we show in Appendix. D, the corrections to $\eta$ for a weakly interacting, normal (unpaired) Fermi gas at low temperatures $(T<\mu)$ are given by (Eq. D25)

$$
\begin{aligned}
& \eta_{0}=\eta(0)\left[1-\frac{31}{84}\left(\lambda k_{F}\right)^{2} \frac{(\nabla \phi)^{2}}{k_{F}^{2} \mu^{2}}+\mathcal{O}\left((\tau \nabla \phi)^{4}\right)\right]=\eta(0)\left[1-\frac{31}{84}\left(\lambda k_{F}\right)^{2} \kappa_{\mathrm{LDA}}^{2}+\mathcal{O}\left((\tau \nabla \phi)^{4}\right)\right] \\
& \eta_{1}=\eta(0)\left[1-\frac{13}{28}\left(\lambda k_{F}\right)^{2} \frac{(\nabla \phi)^{2}}{k_{F}^{2} \mu^{2}}+\mathcal{O}\left((\tau \nabla \phi)^{4}\right)\right]=\eta(0)\left[1-\frac{13}{28}\left(\lambda k_{F}\right)^{2} \kappa_{\mathrm{LDA}}^{2}+\mathcal{O}\left((\tau \nabla \phi)^{4}\right)\right] \\
& \eta_{2}=\eta(0)\left[1-\frac{11}{28}\left(\lambda k_{F}\right)^{2} \frac{(\nabla \phi)^{2}}{k_{F}^{2} \mu^{2}}+\mathcal{O}\left((\tau \nabla \phi)^{4}\right)\right]=\eta(0)\left[1-\frac{11}{28}\left(\lambda k_{F}\right)^{2} \kappa_{\mathrm{LDA}}^{2}+\mathcal{O}\left((\tau \nabla \phi)^{4}\right)\right] \\
& \eta_{3}=0, \eta_{4}=0
\end{aligned}
$$

where $\tau$ is the effective relaxation time.

For the Elliptic mode $\frac{1}{2}\left(\partial_{i} u_{j}+\partial_{j} u_{i}\right)=\frac{1}{2} \alpha_{x}\left(1-\frac{\omega_{x}^{2}}{\omega_{z}^{2}}\right)=V_{x z}$ which probes the viscosity contribution to the stress energy tensor

$$
\sigma_{2_{\alpha \beta}}=2 \eta_{2}\left(V_{\alpha \gamma} b_{\beta} b_{\gamma}+b_{\alpha} V_{\beta \gamma} b_{\gamma}-2 b_{\alpha} b_{\beta} b_{\gamma} b_{\delta} V_{\gamma \delta}\right)
$$


where $b$ is a unit vector along the gradient of the potential. For the Scissor mode, $\frac{1}{2}\left(\partial_{i} u_{j}+\partial_{j} u_{i}\right)=$ $\alpha_{x}=V_{x z}$ which also probes $\eta_{2}$. $\left(\eta_{2}\right.$ is the coefficient that corresponds to the projection operator $M_{2}$ in Eq. D18.)

In both cases (see Appendix. D , $\eta$ is reduced from its value in the absence of the potential, $\eta(0)$, for $\frac{\tau^{2}}{k_{F}^{2}}(\nabla \phi)^{2} \lesssim 1$. To estimate the value of $\tau$ near $z=z_{0}$, we note that for $z \sim z_{0}, T\left(z_{0}\right) \sim$ $0.54 \mu\left(z_{0}\right)$. At this $T, \eta(0) /\left.n\right|_{z_{0}} \sim 1$.

Using the relaxation time approximation and thermodynamic expressions for a weakly interacting Fermi gas to estimate $\lambda$ near $z_{0}$, we obtain (Eq. D26)

$$
\begin{aligned}
\eta(0)\left(z_{0}\right) & =\frac{\left(2 m \mu\left(z_{0}\right)\right)^{\frac{5}{2}} \tau\left(z_{0}\right)}{15 \pi^{2} m} \\
& =\frac{2}{5} n\left(z_{0}\right) \mu\left(z_{0}\right) \tau\left(z_{0}\right)
\end{aligned}
$$

Therefore near $z_{0},\left.\tau\left(z_{0}\right) \sim \frac{5}{2 \mu\left(z_{0}\right)} \frac{\eta(0)}{n}\right|_{z_{0}}$, or,

$$
\begin{aligned}
\lambda\left(z_{0}\right) & =v_{F}\left(z_{0}\right) \tau\left(z_{0}\right) \\
& \left.\sim \frac{k_{F}\left(z_{0}\right)}{m} \frac{5}{2 \mu\left(z_{0}\right)} \frac{\eta(0)}{n}\right|_{z_{0}} \\
& =\left.\frac{5}{4 k_{F}\left(z_{0}\right)} \frac{\eta(0)}{n}\right|_{z_{0}} .
\end{aligned}
$$

(We have just kept the pre-factors of the order of 1 to serve as mnemonics of the derivation of $\lambda$. They have no quantitative significance.)

Therefore, (since $\left.\frac{\eta(0)}{n}\right|_{z_{0}} \sim 1$ from $\frac{\eta}{n}$ data)

$$
\lambda\left(z_{0}\right) k_{F}\left(z_{0}\right)=\left.\frac{5}{4} \frac{\eta(0)}{n}\right|_{z_{0}} \sim 1
$$

The fact that $k_{F}\left(z_{0}\right) \lambda\left(z_{0}\right) \sim 1$ means that the Boltzmann transport calculation shown in Appendix. $\mathrm{D}$ is not quantitatively trustworthy near $z_{0}$. But we hope that two the main qualitative consequences of Eq. 66 survive a more controlled calculation.

1. First, the coefficient of $\kappa_{\mathrm{LDA}}^{2}$ in Eq. 66 is of the order of 1.

2. Second, the sign of the correction term is negative

If true, this would imply that the shear viscosity component $\eta_{x z x z}$ measured using the Elliptic mode or the Scissor mode will reduced by order 1 from its value in isotropic traps, if $\omega_{z} \gtrsim 2 \pi \times 77000$ rads/s (Table. II). 
One might be concerned that for $\omega_{z} \sim 2 \pi \times 77000 \mathrm{rads} / \mathrm{s}$, our conclusions in the previous section about $\delta z / z_{0}$ will be violated because of the violation of LDA. In the absence of more concrete information on these coefficients we can not assure this will not happen. We simply note that if the coefficient $c_{1}$ in Eq. 62 is of the order of 1 (which it is at $T \ll \mu$, but may be larger for $\left.T \sim 0.54 \mu\left(z_{0}\right)\right)$ then there is a regime where the corrections to the thermodynamics due to LDA is small, but the reduction in transport coefficients is substantial.

\section{CONCLUSIONS}

We present a concrete realization of a system of ultra-cold Fermi gases at unitarity, in an anisotropic trap, which may show significant reduction in the viscosity compared to its value in isotropic traps. Given that the value of the isotropic viscosity has been measured to be few times the KSS bound in this system, it presents a candidate setup to observe a shear viscosity smaller than the KSS bound when it is subjected to an anisotropic driving force.

The anisotropic force is obtained by placing the system in an anisotropic trap. The trapping potential is harmonic, Eq. 6, and characterized by three angular frequencies, $\omega_{x}, \omega_{y}, \omega_{z}$. We consider an anisotropic situation where $\omega_{z} \gg \omega_{x}, \omega_{y}$, so that the trapping potential is much stronger in the $z$ direction. For simplicity, we also take $\omega_{x}=\omega_{y}$ so that the system preserves rotational invariance in the $x-y$ plane. For some of the discussion below we can neglect the effects of the trapping potential in the $x, y$ directions characterized by $\omega_{x}, \omega_{y}$.

We work in conventions where $k_{B}=\hbar=1$. There are three energy scales $T, \mu, \omega_{z}$ and two dimensionless ratios $T / \mu$ and $\omega_{z} / \mu$ which then characterize the system. The $\operatorname{Li}_{6}$ atoms have a mass $m$, using this parameter, any of the energy scales can be converted to a length scale, $L=\frac{1}{\sqrt{2 m E}}$.

Based on the behavior seen quite generically in gravity systems we identify five criterion (Sec. II ) which when met could plausibly lead to a decrease in the value of some components of the viscosity tensor (the spin one components). These are summarized towards the end of Sec. II. On studying the superfluid equations we identify two modes which are sensitive to these components of the viscosity tensor. One of these is the scissor mode which has already been studied experimentally in some detail. By taking reasonable values for the parameters- $T, \mu, \omega_{z}, \omega_{x}, \omega_{y}$, which are in the experimentally accessible range, Ref. [22], we find that all the five criteria can be met. Furthermore, we find that the resulting energy and damping rate of this energy, from which the viscosity can be extracted, lie within the range of values which are measured by experiments currently being done on cold atom systems, in particular on $\mathrm{Li}_{6}$ unitary Fermi gas systems, Ref. [49]. For example, 
for $\mu=10 \mu \mathrm{K}, \omega_{z} \sim 2 \pi \times 77000 \mathrm{rads} / \mathrm{s}$, and $T=\frac{T_{c}}{2}\left(T_{c}=0.4 \mu\right)$ we find that the anisotropy, as measured by the parameter $\kappa_{L D A}$, Eq. 43 , is of order unity and therefore significant. At these extreme values of anisotropy our theoretical calculation, strictly speaking, do not apply, but a reasonable extrapolation suggests that the maximum total energy is of the order of $10^{-17}$ joules which corresponds to the angular amplitude of the scissor mode of about $24^{\circ}$ which is within the experimental range of [49]. The damping time $\tau_{0}$ is of the order of $10^{-2}$ seconds, which is roughly ten times longer than the observed amplitude damping time that has been accurately measured in the experiments on ultracold Fermi gases [49].

While the system is certainly close to being two-dimensional when $\kappa_{L D A} \sim 1$ and $z_{\text {trap }} \sim 5.4 k_{F}^{-1}$ (this corresponds to $\mu / \omega_{z} \sim 2.7$ ) is on the small side, the effect of small viscosity can already set in when $\kappa_{L D A}$ is somewhat smaller than unity. We illustrate this with concrete quantitative examples below.

For concreteness, let us consider traps where we fix $T / T_{c}=1 / 2\left(T_{c}=0.4 \mu\right.$, where $\mu$ is the chemical potential at the center of the trap) and change $\omega_{z}$. Further, for concreteness, we set the overall scale by $\mu=10 \mu \mathrm{K}$. Considering first a representative trap geometry where the shear viscosity tensor is locally isotropic to a large accuracy, we take $\omega_{z}=0.048 \mu$ (corresponding to $\omega_{z}=2 \pi \times 10^{4} \mathrm{~Hz}$ which is typical), for which $\kappa_{\mathrm{LDA}}=0.13$. The fractional reduction in the shear viscosity for this value of $\omega_{z}$, taking $c_{2}$ to be its Boltzmann transport value $11 / 28$ is

$$
\frac{\Delta \eta}{\eta} \approx-\frac{11}{28}\left(\kappa_{\mathrm{LDA}}\right)^{2}=-0.7 \%
$$

which is a small reduction in the shear viscosity and may not be even measurable above measurement errors. At the other extreme we considered, $\omega_{z}=\frac{\mu}{2.7}$ (corresponding to $\omega_{z}=2 \pi \times 77.16 \mathrm{kHz}$ ), for which $\kappa_{\mathrm{LDA}}=1$ and the fractional reduction is

$$
\frac{\Delta \eta}{\eta} \approx-\frac{11}{28}\left(\kappa_{\mathrm{LDA}}\right)^{2}=-39 \%
$$

which is very large. However, in this extreme limit $\left(\omega_{z}=\frac{\mu}{2.7}\right)$ only the lowest $2-3$ Landau levels are occupied and the dynamics may be approximately two dimensional. Now consider an intermediate value, say $\omega_{z}=0.9 T=0.18 \mu$ for which $\kappa_{L D A}=0.48<1$. This gives a correction

$$
\frac{\Delta \eta}{\eta} \approx-9 \%
$$


which — while not large — is still substantial. More generally, the criterion for confinement in the $z$ direction is

$$
\omega_{z} \gtrsim \max (\Delta, T)
$$

since both $T$ and pairing allow for excitations between the harmonic oscillator levels. At these extreme values, where the inequality above is met, our approximations do break down, (shell effects become important as $\omega_{z} \gtrsim T$, which is another way of saying that confinement in the $z$ direction becomes strong). For $\omega_{z}=\frac{\mu}{2.7}, \omega_{z}=1.85 T$ and indeed confinement in the $z$ direction is too strong. But, as illustrated by the cases above, by taking $\omega_{z}$ a factor of 2 or 3 smaller ( say $\omega_{z}=0.9 T$ that was chosen above for illustration ${ }^{6}$ ) than the extreme limit, one can measure the tendency of the spin one component of the viscosity to decrease from its lowest value observed in ultra-cold Fermi gases. In an optimistic scenario where $c_{2}$ is larger in magnitude than the approximate value of $11 / 28$ in the Boltzmann transport approximation, the reduction will be even more substantial. Let us also point out that comparing with Ref. [81] the typical values of $\omega_{z} / E_{F}$ in the paper is about 80 and the value of $\omega_{z} / T$ is 120 . In that case, the trap is truly 2 dimensional as opposed to when $\omega_{z} / T \sim 0.9$.

Thus, for smaller values of anisotropy, the theoretical estimates are more reliable and suggest that the different viscosity tensor components should have a fractional difference given in terms of $\kappa_{L D A}$ by Eq. 66. This tendency of the viscosity to decrease should already be measurable at more moderate values of the anisotropy.

Our proposal is the first proposal to measure parametrically suppressed anisotropic viscosity components in ultra-cold Fermi gases. Our proposal is different from the discussion of anisotropic hydrodynamics in Ref. [57] since we are demanding that hydrodynamics be a good description (in the sense of Eq. 15) in the regime which dominantly contributes to the dissipation of the fluid dynamics modes.

Future theoretical work can improve upon our proposal in several ways. First, our estimate of the corrections to the shear viscosity components due to the potential (Eq. 66) was based on a relaxation time treatment of the Boltzmann equation. For strongly interacting fermions, this is not a good approximation and a more rigorous calculation of the anisotropy corrections is

\footnotetext{
${ }^{6}$ The deviations from LDA due to shell effects for unpaired fermions was calculated in Ref. 80. A naive application of the results of Ref. [80] suggests that for our trap with $\omega_{z}=0.18 \mu$, the corrections to the number density is about $15 \%$ at $T=4 T_{c} / 5$ near the region relevant for our purposes. Note however, that pairing suppresses LDA violations $([2,77])$ and we expect the corrections to be much smaller in the relevant region.
} 
desirable. This will require calculating transport properties in a strongly coupled theory without a gravitational dual, in the presence of a background potential: a formidable challenge. Second, we have focused on the region that dominantly contributes to the dissipation. In particular we have neglected the contributions from the tail of the cloud. While this is presumably small, it would be nice to establish this by solving the Boltzmann transport equations in this dilute regime.

It is also worth noting that while the cold-atom system proposed here shares many features with those discussed in Ref. [42, 45], it also has some differences. First, in equilibrium the stress energy tensor is not invariant under translations even for a linear potential. Rather the density decreases with increasing $z$, but the driving force is proportional to the gradient of the potential $\phi(\mathbf{r})$ (see Eq. 3) as in Ref. [42, 45]. Second, in addition to energy-momentum, the cold-atom system features another conserved quantity: the particle number. Consequently the system is locally characterized by two thermodynamic variables $T$ and $\mu$ rather than just $T$. It would also be interesting to further study the behavior of viscosity in gravitational systems which correspond to anisotropy driven strongly coupled systems with a finite chemical potential. The examples in Ref. [42, 45] did not have a finite chemical potential, for some discussion of anisotropic gravity systems with a chemical potential see Ref. [44, 82]. As a first step, we have analyzed a weakly coupled system with a linear varying potential in Appendix. $\mathrm{D}$ and find that the viscosity does become anisotropic in this case.

However, there is no reason to wait for these theoretical advances. The central point of this paper is that there is already enough motivation, based on the behavior quite generically seen in gravitational systems, to suggest that some components of the viscosity tensor in anisotropic strongly coupled systems might well become small, making $\eta / s$ for these components potentially even smaller than the KSS bound, $1 / 4 \pi$. Such a decrease in the viscosity might well happen in cold atom systems, for example the unitary fermi gas, which are experimentally well studied. As argued above, the range of values involved for temperature, chemical potential and angular frequencies are well within the experimental regime for such a system, and the scissor mode which is sensitive to the relevant components of the viscosity has already been realised experimentally in them. Further, the resulting values for the energy and the damping time from which the viscosity can be extracted lie in the experimentally accessible range which has already been achieved.

We hope our experimental colleagues in the cold atoms community will take note of these results, and implore them to carry out a study of viscosity in anisotropic traps. 


\section{ACKNOWLEDGMENTS}

We thank D. D. Ofengeim and in particular D. G. Yakovlev for sharing their notes on the calculation of the various components of viscosities in the presence of the magnetic field. We especially thank M. Randeria for sharing his valuable comments and insights. We also acknowledge conversations with K. Damle, S. Gupta, S. Jain, N. Kundu, G. Mandal, S. Minwalla, T. Schäfer, R. SenSarma and N. Trivedi. SPT acknowledges support from the J. C. Bose fellowship DST, Government of India. We acknowledge support from the DAE, Government of India. Most of all we thank the people of India for supporting our research.

\section{Appendix A: More details on the results of shear viscosity from gravity}

In Ref. [45], several anisotropic theories in $3+1$ dimensional space-time (the boundary with coordinates $(t, x, y, z))$, which are dual to a gravitational theory living in $4+1$ dimensional spacetime (the bulk with an additional coordinate $u$ ) were studied. Isotropy was broken by considering states where some of the fields have a background value that depended on some of the spatial coordinates $x, y, z$, explicitly breaking rotational symmetry between them.

All the examples studied in Ref. [45] share the common feature that the force responsible for breaking isotropy in the boundary theory is translation invariant as we shall explain via an example below.

Ref. [45] built on the results of Ref. [42, which studied a simple system consisting of a linearly varying dilaton. The dilaton field $\phi$ couples to the graviton in the bulk via the Lagrangian

$$
S=\frac{1}{16 \pi G} \int d^{5} x \sqrt{g}\left[R+12 \Lambda-\frac{1}{2} \partial_{\mu} \phi \partial^{\mu} \phi\right]
$$

where $G$ is Newton's constant in 5 dimensions and $\Lambda$ is a cosmological constant. The boundary theory in the absence of anisotropy is a $3+1$ dimensional conformal field theory.

In this system we can clarify what we mean by saying that the driving force is constant. The dilaton field in the background solution here has the profile

$$
\phi(t, x, y, z)=\rho z
$$

Clearly this choice of the background singles out the $z$ direction, breaking isotropy. In the presence 
of the dilaton the conservation equations for the stress tensor get modified to be,

$$
\partial_{\mu} T^{\mu \nu}=\langle O\rangle \partial^{\nu} \phi
$$

where $O$ is the operator dual to the field $\phi$. The right hand side arises because the varying dilaton results in a driving force on the system. We see that a linear profile results in a constant value for $\partial^{\nu} \phi$ and thus a constant driving force.

Let us also mention that in this example, on the gravity side the linearly varying dilaton gives rise to a translationally invariant stress tensor and thus a black brane solution which preserves translational invariance. This corresponds to the fact that in the field theory the equilibrium stress tensor features only derivatives of $\phi$ and is thus space-time invariant.

We shall see that the cold-atom system we consider will not be invariant under translations in equilibrium. However the equations of hydrodynamics (Eq. B3) in the presence of a driving force associated with a space varying potential look similar to Eq. A3, where the operator $O$ in the cold-atom system corresponds to the density, and the driving force is proportional to the gradient of the potential $\phi(\mathbf{r})$.

The example considered in Ref. [42] also shares the property that an $S O(2,1)$ residual Lorentz symmetry survives, at zero temperature, after breaking isotropy. This residual Lorentz symmetry corresponds to the $t, x, y$ directions in the boundary theory. Fluid mechanics corresponds to the dynamics of the Goldstone modes associated with the boost symmetries of this residual Lorentz group, which are broken at finite temperature.

In a general system the viscosity $\eta$ is a fourth order tensor under rotations relating the deviation of the stress-energy tensor from its equilibrium value, to the velocity gradient. If the local fluid velocity is $\mathbf{v}=\left(v_{x}, v_{y}, v_{z}\right)$, we have

$$
\delta T^{i j}=\eta^{i j k l} \frac{1}{2}\left(\partial_{k} v_{l}+\partial_{l} v_{k}\right)
$$

Since we are only considering the effects of the shear components,

$$
\eta^{i j k l} \delta_{k l}=0
$$

In the example in Ref. [42, with dilaton profile given by Eq. A2, the viscosity components that become small correspond to the $\eta^{x z x z}, \eta^{y z y z}$ components of the viscosity tensor. In the subsequent 


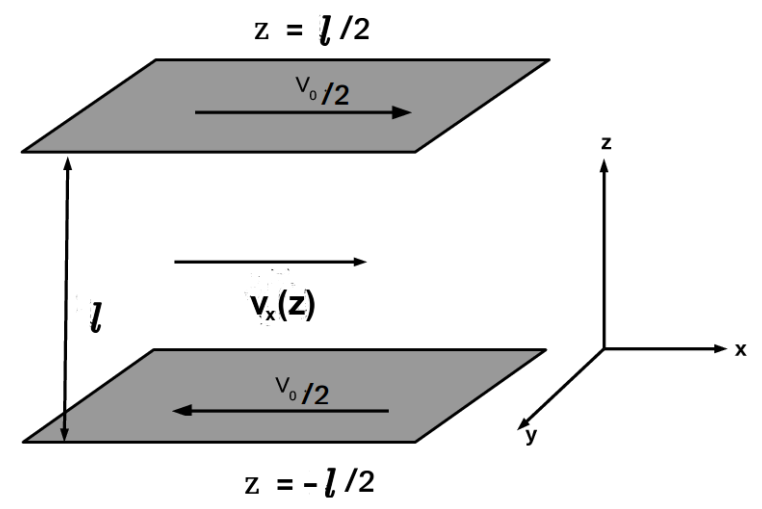

FIG. 8: Fluid flow between two parallel plates. For $\phi=\rho z$ the driving force is in the $z$ direction and is proportional to $\rho$. Parametrically small values of the viscosity (Eq. A12 govern the dynamics for flows in the $x$ (or $y$ ) direction with a gradient in the $z$ direction (for Eg. $v_{x}=v_{0} z$ ).

discussion we shall use an abbreviated notation,

$$
\eta^{x z x z}=\eta_{x z}, \eta^{y z y z}=\eta_{y z}
$$

In the gravity description these components correspond to perturbations of the metric which carry spin 1 with respect to the surviving $S O(2,1)$ residual Lorentz symmetry.

A fluid flow configuration where the frictional force (and therefore the resulting dissipation) is governed by a spin 1 viscosity component arises as follows. Consider the fluid enclosed between $([45,53])$ two parallel plates separated along $z$ axis by a distance $L$ with the top plate moving with a speed $v_{0} / 2$ along $x$ direction while the lower plate moves with a speed $v_{0} / 2$ along $-x$ direction, see Fig.8.

The resulting steady state solution of the Navier Stokes equation, even for the anisotropic case, is remarkably simple, with

$$
v_{y}=0, v_{z}=0
$$

the temperature $T$ being a constant, and $v_{x}$ being a linear function of $z$

$$
v_{x}=\frac{v_{0}}{L} z, z \in(-L / 2, L / 2)
$$

( we have chosen coordinates so that $z=0$ lies at the midpoint between the plates). A constant force per unit area is exerted by the fluid on both the upper and lower plates, $T^{x z}=\eta_{x z} \partial_{z} v_{x}$, 
in this solution (we are compactly writing $\eta_{x z x z}$ as $\eta_{x z}$ ). This frictional force retards the relative motion of the plates and must be counteracted by an equal and opposite force acting on both plates externally to sustain the steady state solution ${ }^{7}$. We also note that for this solution, in the gravity theory under discussion, hydrodynamics is valid as long as the velocity gradient $\frac{v_{0}}{L}$ is small compared to the temperature $T$.

Using results from the gauge-gravity duality [11] it was shown in Ref. [45] quite generally that the viscosity component $\eta_{x z}$ behaves like

$$
\frac{\eta_{x z}}{s}=\left.\frac{1}{4 \pi} \frac{g_{x x}}{g_{z z}}\right|_{u=u_{h}}
$$

where $\left.g_{x x}\right|_{u=u_{h}},\left.g_{z z}\right|_{u=u_{h}}$ refer to the components of the background metric evaluated at the horizon which we denote by $u_{h}$. ' $s$ ' refers to the entropy density which in the bulk picture corresponds to the area of the event horizon.

In the isotropic case the ratio $\left.\frac{g_{x x}}{g_{z z}}\right|_{u=u_{h}}$ is unity and we see that the KSS result is obtained. However, in anisotropic cases this ratio can become very different from unity and in fact much smaller, leading to the parametric violation of the KSS bound, where the relevant dimensionless parameter is the ratio of the strength of the anisotropic interaction and an appropriate microscopic energy scale of the system.

The general result Eq. A9, for the behavior of the spin 1 shear viscosity components $\eta_{x z}=\eta_{y z} \equiv$ $\eta_{\perp}$ was studied in the example of Ref. 42] for two cases - one in the low anisotropy regime and the other in the high anisotropy regime. In this example, there are two scales of interest, $\rho$, which enters in the dilaton profile, Eq. A2 and determines the anisotropy, and the temperature $T$ (while this theory does not have quasi-particles at finite $T$, one can roughly think of the mean free path as being of the order of $1 / T)$. Whether the anisotropy is large or small is determined by the ratio $\rho / T$ which is dimensionless. Simple results can be obtained in the limit of low and high anisotropy which correspond to $\rho / T \ll 1$ and $\rho / T \gg 1$ respectively.

For the spin 1 component of the shear viscosity $\eta_{x z}=\eta_{y z} \equiv \eta_{\perp}$ the results are as follows:

1. Low anisotropy regime $(\rho / T \ll 1)$ :

$$
\frac{\eta_{\perp}}{s}=\frac{1}{4 \pi}-\frac{\rho^{2} \log 2}{16 \pi^{3} T^{2}}+\frac{\left(6-\pi^{2}+54(\log 2)^{2}\right) \rho^{4}}{2304 \pi^{5} T^{4}}+\mathcal{O}\left[\left(\frac{\rho}{T}\right)^{6}\right]
$$

\footnotetext{
${ }^{7}$ It is interesting to note that this solution for $\mathbf{v}$ is essentially the same as that of an isotropic fluid.
} 
We see that a small anisotropy at order $(\rho / T)^{2}$ already reduces this component of the viscosity and makes it smaller than the KSS bound. In the limit of zero anisotropy, we recover the KSS bound

$$
\frac{\eta_{\perp}}{s} \rightarrow \frac{1}{4 \pi}
$$

We also note that the driving force in the conservation equation for the stress tensor (Eq. A3) is proportional to $\nabla \phi \sim \rho$ (Eq. A2) and the analogue of the mean free path is T. Thus the corrections go like $\frac{(\nabla \phi)^{2}}{T^{2}}$.

2. High anisotropy regime $(\rho / T \gg 1)$ :

$$
\frac{\eta_{\perp}}{s}=\frac{8 \pi T^{2}}{3 \rho^{2}}
$$

We see that in this limit the ratio can be made arbitrarily small, with $\frac{\eta_{\perp}}{s} \rightarrow 0$, as $T \rightarrow 0$ keeping $\rho$ fixed. 8

In contrast the $\eta_{x y x y}$ component (which couples to a spin 2 metric perturbation) was found to be unchanged from its value in the isotropic case,

$$
\frac{\eta_{x y x y}}{s}=\frac{1}{4 \pi}
$$

and thus continues to meet the KSS bound.

\section{Appendix B: Derivation of hydrodynamic modes}

In this appendix, we will first show that the Elliptic mode and the Scissor mode satisfy the equations of superfluid hydrodynamics in the presence of a harmonic trap. There are viscous corrections to the hydrodynamic equations, but we work in a limit where viscous corrections are small and therefore the solutions to the ideal hydrodynamics can be used to calculate the energy loss rate due to viscosity in a perturbative manner.

\footnotetext{
${ }^{8}$ In this regime $\eta_{\perp} \sim \frac{T^{4}}{\rho}$ and $s \sim T^{2} \rho$, whereas for the isotropic case $(\rho=0) \eta_{\perp} \sim T^{3}$ and $s \sim T^{3}$. Thus we see that for $T \ll \rho, \eta_{\perp}$ is smaller than its value in the isotropic case while $s$ is bigger, resulting in the parametric violation in Eq. A12.
} 


\section{Equations of superfluid hydrodynamics}

Neglecting viscosity, the superfluid equations are given by the conservation laws of entropy, mass (particle number), momentum and an additional equation for the superfluid velocity. In the presence of the external potential $\phi(\mathbf{r})$ they are listed below :

$$
\begin{aligned}
& \frac{\partial(\rho s)}{\partial t}+\nabla \cdot\left(\rho s \mathbf{v}_{n}\right)=0, \\
& \frac{\partial \rho}{\partial t}+\nabla \cdot \mathbf{g}=0, \\
& \frac{\partial g_{i}}{\partial t}+\nabla_{j} \Pi_{i j}=-n \nabla \phi(\mathbf{r}), \\
& \frac{\partial \mathbf{v}_{s}}{\partial t}=-\nabla\left(\frac{\mathbf{v}_{s}^{2}}{2}+\frac{\phi(\mathbf{r})}{m}+\frac{\mu(\mathbf{r})}{m}\right) .
\end{aligned}
$$

Here $\rho$ is the total mass density (where $\rho_{n}$ and $\rho_{s}$ are the normal and superfluid mass density of the system and the total mass density $\left.\rho=\rho_{n}+\rho_{s}\right)$. We have not written out the dependence of the velocity on position and time. $\mu(\mathbf{r})$ can be thought of as the local chemical potential. $n$ (not in the subscript) denotes the total number density (which is related to the total mass density $\rho$ via the relation $\rho=m n)$, $\mathbf{g}$ is the momentum density, and $\Pi_{i j}$ is the stress tensor, given as follows

$$
\begin{aligned}
\mathbf{g} & =\rho_{n} \mathbf{v}_{n}+\rho_{s} \mathbf{v}_{s}, \\
\Pi_{i j} & =P \delta_{i j}+\rho_{n} \mathbf{v}_{n, i} \mathbf{v}_{n, j}+\rho_{s} \mathbf{v}_{s, i} \mathbf{v}_{s, j} .
\end{aligned}
$$

Let us note that the equation for energy conservation can be derived from the set of equations above, and is not an additional independent constraint.

Altogether there are 8 equations above and they can be solved for the 8 independent variables - 6 components of $\left(\mathbf{v}_{\mathbf{s}}, \mathbf{v}_{\mathbf{n}}\right)$ and $T, \mu(\mathbf{r})$. We can then express all thermodynamic variables as functions of $(T, \mu(\mathbf{r}))$ like $P(T, \mu(\mathbf{r})), s(T, \mu(\mathbf{r}))$ etc. In the trap geometries we consider, the center of the trap is superfluid and the outer trap is in the normal phase. The equations for a normal fluid can be obtained by simply substituting $\rho_{s}=0$ and ignoring Eq. B4.

Let us first look at the equilibrium situation $\mathbf{v}_{\mathbf{n}}=\mathbf{v}_{\mathbf{s}}=\mathbf{0}$ in the absence of external potential $\phi$. Eqns. B1, B2, B3, B4 are satisfied with $\mu(\mathbf{r})$ and $P$ spatially constant.

Before we consider the effects of an external potential let us also note that the pressure and number density in the absence of the trap, which we denote as $P_{\phi=0}, n_{\phi=0}$ respectively, satisfy the 
Gibbs-Duhem relation

$$
\frac{\partial P_{\phi=0}}{\partial \mu}=n_{\phi=0}
$$

In the presence of the external potential $\phi(\mathbf{r})$ with $\mathbf{v}_{s}=\mathbf{v}_{n}=0$, only Eq. B3 and Eq. B4 changes. Eq. B4 is satisfied by taking

$$
\mu(\mathbf{r})=\mu-\phi(\mathbf{r})
$$

where $\mu$ is a global constant that determines the total number of particles in the system. Eq. B3 in the presence of $\phi(\mathbf{r})$ becomes

$$
\partial_{i} P(\mathbf{r})=-n \partial_{i} \phi(\mathbf{r})
$$

This is consistent with the replacement $\mu(\mathbf{r}) \rightarrow \mu-\phi(\mathbf{r})$ if we take the pressure $P$ at a point $\mathbf{r}$ in the presence of the trap to be equal to $P_{\phi=0}(T, \mu-\phi(\mathbf{r}))$ and the number density to be $n_{\phi=0}(T, \mu-\phi(\mathbf{r}))$. This follows from Eq. $\mathrm{B} 6$, since $\partial_{i} P=-\frac{\partial P_{\phi=0}}{\partial \mu} \partial_{i} \phi=-n_{\phi=0} \partial_{i} \phi$. This is also known as LDA (Local Density Approximation). Generally LDA corresponds to the conditions,

$$
f(\mu(\mathbf{r}), T):=f_{\phi=0}(\mu-\phi(\mathbf{r}), T)
$$

where $f$ is $P, n, \rho$ or $s$. In all the subsequent discussions, a subscript 0 indicates that the conditions for LDA are valid in equilibrium. Note that in equilibrium $T$ is a constant.

\section{Scissor mode solution to linear order}

First we look for solutions of the form

$$
\mathbf{v}_{n}=\mathbf{v}_{s}=\mathbf{v}
$$

and $\nabla \times \mathbf{v}=\mathbf{0}$. We restrict ourselves to small velocities and linearize the above equations. For the scissor mode we see from Eq. 7 and Eq. 9 that $\mathbf{v}$ is given by

$$
\mathbf{v}=\alpha e^{i \omega t}(z \hat{x}+x \hat{z})
$$


where $\alpha=\alpha_{x}=\alpha_{z}$ is a constant. We will solve the equations to linear order in $\alpha$.

Let us first explore Eq. B4. Out of equilibrium $(\mathbf{v} \neq 0), \mu(\mathbf{r})$ has an extra correction associated with $\mathbf{v}$,

$$
\mu(\mathbf{r})=\mu-\phi(\mathbf{r})+\epsilon(\mathbf{r}, t) .
$$

Eq. B4 then gives

$$
\epsilon=-\alpha m x z i \omega e^{i \omega t}
$$

Once we are out of equilibrium, we will see that the remaining equations are self consistently solved by letting

$$
f_{\phi \neq 0}(\mu(\mathbf{r}), T):=f_{\phi=0}(\mu-\phi(\mathbf{r})+\epsilon(\mathbf{r}, t), T)
$$

where $f$ is $P, n, \rho$ or $s$.

The mass and momentum conservation equations, with the condition Eq. (B10), give

$$
\begin{aligned}
\frac{\partial \rho}{\partial t}+\nabla \cdot(\rho \mathbf{v}) & =0 \\
\rho \frac{\partial \mathbf{v}}{\partial t}+\rho(\mathbf{v} \cdot \nabla) \mathbf{v} & =-\nabla P-n \nabla \phi
\end{aligned}
$$

where $\phi(\mathbf{r})$ is the external potential and $\rho$ is the total mass density $\left(\rho_{n}+\rho_{s}\right)$. Linearizing these equations to order $\alpha^{9}$ using Eq. B14 we get,

$$
\begin{aligned}
& \frac{\partial \rho_{0}}{\partial \mu} \frac{\partial \epsilon}{\partial t}+\nabla \cdot\left(\rho_{0} \mathbf{v}\right)=0 \\
& \rho_{0} \frac{\partial \mathbf{v}}{\partial t}=-\nabla\left(\frac{\partial P_{0}}{\partial \mu} \epsilon\right)-\left(\frac{\partial n_{0}}{\partial \mu} \epsilon\right) \nabla \phi .
\end{aligned}
$$

Using $\partial_{i} \rho_{0}=-\frac{\partial \rho_{0}}{\partial \mu} \partial_{i} \phi$ and using the fact that for the modes we consider in this paper $\nabla \cdot \mathbf{v}=0$ we get from Eq. B17

$$
\frac{\partial \epsilon}{\partial t}-\partial_{i} \phi \mathbf{v}_{i}=0
$$

Plugging in the harmonic potential and the solution Eq. B13, we find that the above equation is

\footnotetext{
${ }^{9}$ Note that $\epsilon$ in Eq. B13 is of order $\alpha$
} 
solved by the Scissor mode which satisfies the condition, Eq. 9. Now taking time derivative of the

Euler equation Eq. B18 and using Eq. B17 in the second term on R.H.S of Eq. B18 and $\frac{\partial P_{0}}{\partial \mu}=n_{0}$ (total number density at equilibrium),

$$
\begin{aligned}
& \rho_{0} \frac{\partial^{2} \mathbf{v}_{i}}{\partial t^{2}}=-\partial_{i}\left(n_{0} \frac{\partial \epsilon}{\partial t}\right)+\partial_{j}\left(n_{0} \mathbf{v}_{j}\right) \partial_{i} \phi \\
& \Rightarrow \rho_{0} \frac{\partial^{2} \mathbf{v}_{i}}{\partial t^{2}}+n_{0} \partial_{i}\left(\frac{\partial \epsilon}{\partial t}\right)=-\partial_{i} n_{0}\left(\frac{\partial \epsilon}{\partial t}\right)+\partial_{j} n_{0} \mathbf{v}_{j} \partial_{i} \phi \\
& \Rightarrow \rho_{0} \frac{\partial^{2} \mathbf{v}_{i}}{\partial t^{2}}+n_{0} \partial_{i}\left(\frac{\partial \epsilon}{\partial t}\right)=\frac{\partial n_{0}}{\partial \mu} \partial_{i} \phi\left(\frac{\partial \epsilon}{\partial t}\right)-\frac{\partial n_{0}}{\partial \mu} \partial_{j} \phi \mathbf{v}_{j} \partial_{i} \phi .
\end{aligned}
$$

We see from Eq. B19 that the RHS of the above equation vanishes. For the scissor mode, it follows from Eq. 9 and Eq. B13 that the LHS also vanishes, and thus the equation is met.

For the time dependent scissor mode, the mass conservation equation is

$$
\frac{\partial \rho}{\partial t}+\nabla \cdot(\rho \mathbf{v})=0
$$

for $\mathbf{v}_{s}=\mathbf{v}_{n}=\mathbf{v}$.

Starting with Eq. B1 and using Eq. B21 we get

$$
\frac{\partial s}{\partial t}+\mathbf{v} \cdot s=0
$$

Assuming that the entropy is of the form $s(\mu-\phi(\mathbf{r})+\epsilon(\mathbf{r}, t))$ as given in Eq. B14 and linearizing in $\alpha$ we get

$$
\frac{\partial s_{0}}{\partial \mu} \frac{\partial \epsilon}{\partial t}-\frac{\partial s_{0}}{\partial \mu} \partial_{i} \phi \mathbf{v}_{i}=0
$$

This equation is valid when Eq. B19 is met. Hence we find that the ansatz Eq. B14 with Eq. B13 meets all the equations self consistently.

\section{Elliptic mode solution to linear order}

Next we verify that the Elliptic mode, Eq.8, solves the superfluid equations to linear order in the velocity. Note that this mode is a stationary solution $(\omega=0)$. Like in the previous case we take $T$ to be a constant in this mode. Note that in this solution $\mathbf{v}_{n}$ has a non-zero curl, $\nabla \times \mathbf{v}_{n} \neq 0$, and therefore in the absence of vortices $\mathbf{v}_{s} \neq \mathbf{v}_{n}$. We will denote $\mathbf{v}_{n}=\mathbf{v}$ below. 
We start with Eq. B4. Since $\mathbf{v}_{s}=0$ in this mode, we see that this equation is met if

$$
\mu(r)=\mu-\phi(r)
$$

where $\mu$ on the RHS is an $\mathbf{r}$ independent constant.

Next, with $\mathbf{v}_{s}=0$ the mass and momentum conservation equations simplify to

$$
\begin{aligned}
\frac{\partial \rho}{\partial t}+\nabla \cdot\left(\rho_{n} \mathbf{v}\right) & =0 \\
\frac{\partial\left(\rho_{n} \mathbf{v}_{\mathbf{i}}\right)}{\partial t}+\nabla_{j}\left(\rho_{n} \mathbf{v}_{\mathbf{i}} \mathbf{v}_{\mathbf{j}}\right) & =-\nabla_{i} P-n \nabla_{i} \phi .
\end{aligned}
$$

The time derivatives in these equations can be dropped. The Euler equation, Eq. B26, is met to order $\mathbf{v}$ if $P$ and $n$ take their form in the LDA approximation, Eq. B9. We will also assume that the other thermodynamic values, $\rho_{n}, s$ take this LDA form and denote them with a subscript 0. Using the fact that $\nabla \cdot \mathbf{v}=0$, the other equation, Eq. B25, becomes,

$$
\nabla \cdot\left(\rho_{0 n} \mathbf{v}\right)=0 \Rightarrow-\frac{\partial \rho_{0 n}}{\partial \mu} \partial_{i} \phi \mathbf{v}_{i}=0
$$

where we have used the ansatz Eq. B9 for the mass density of the normal component. For our mode $\alpha_{x} z \hat{x}+\alpha_{z} x \hat{z}$ with $\alpha_{z}=-\frac{\omega_{x}^{2}}{\omega_{z}^{2}} \alpha_{x}$ (see Eq. 8) one can easily check that

$$
\partial_{i} \phi \mathbf{v}_{i}=0
$$

so that this equation is satisfied.

Finally, the entropy conservation equation (after replacing $\rho, s$ by their LDA values) becomes

$$
\nabla \cdot\left(\rho_{0} s_{0} \mathbf{v}\right)=0
$$

Using the fact that our mode is free of divergence, and $\rho_{0} s_{0}$ is a function of $\mu-\phi(\mathbf{r})$, we see that this equation is also met when Eq. B28 is satisfied.

It is interesting to note that the fact that the Elliptic mode and the Scissor mode also solve the equations of one component fluid mechanics in the normal phase. Since the temperature is a constant in these modes, and the chemical potential varies as given in Eq. B7, up to possible corrections of order $\epsilon$, Eq. B12, as one moves from the center of the trap to its edges the ratio $\mu(r) / T$ becomes smaller and the system will transit from the superfluid to normal phase. The 
solutions we have found above, for both modes, will continue to hold in such situations as well.

\section{Appendix C: Ideal hydrodynamic modes}

In this section we contrast the modes discussed in Sec. III A with the breathing modes discussed in Ref. 21] in normal fluids.

We start with the linearized continuity and Euler equations for a fluid with a polytropic equation of state, which can be used to derive the following equation valid for ideal fluid dynamics for the normal component [21,

$$
m \frac{\partial^{2} \mathbf{v}}{\partial t^{2}}=-\gamma(\nabla \cdot \mathbf{v})(\nabla \phi(\mathbf{r}))-\nabla(\mathbf{v} \cdot \nabla \phi(\mathbf{r}))
$$

As shown in Ref. [21] breathing modes can be obtained by considering a scaling ansatz $v_{i}=$ $a_{i} x_{i} \exp (i \omega t)$ (no sum over $i$ ). Substituting in Eq. C1 one obtains an eigenequation

$$
\left(2 \omega_{j}^{2}-\omega^{2}\right) a_{j}+\gamma \omega_{j}^{2} \sum_{k} a_{k}=0
$$

This is a simple linear equation of the form $M a=0$. Non-trivial solutions correspond to $\operatorname{det}(M)=$ 0 .

In the case of a trapping potential with axial symmetry, $\omega_{1}=\omega_{2}=\omega_{0}, \omega_{3}=\lambda \omega_{0}$, we get $\omega^{2}=2 \omega_{0}^{2}$ and [50, 67, 83]

$$
\begin{aligned}
\omega^{2}= & \omega_{0}^{2}\left\{\gamma+1+\frac{\gamma+2}{2} \lambda^{2}\right. \\
& \left. \pm \sqrt{\frac{(\gamma+2)^{2}}{4} \lambda^{4}+\left(\gamma^{2}-3 \gamma-2\right) \lambda^{2}+(\gamma+1)^{2}}\right\}
\end{aligned}
$$

In the unitarity limit $(\gamma=2 / 3)$ and for a very asymmetric trap, $\lambda \rightarrow 0$, the eigen-frequencies are $\omega^{2}=2 \omega_{0}^{2}$ and $\omega^{2}=(10 / 3) \omega_{0}^{2}$. The mode $\omega^{2}=(10 / 3) \omega_{0}^{2}$ is a radial breathing mode with $\mathbf{a}=(a, a, 0)$ and the mode $\omega^{2}=2 \omega_{0}^{2}$ corresponds to a radial quadrupole $\mathbf{a}=(a,-a, 0)$.

Here we consider a different class of modes, with the scaling form Eq. C4 (since $x$ and $z$ are exchanged, they are "twisted"). The eigen-equations are now given by Eq.C5. It has two solutions,

$\omega=0$ and $\omega=\sqrt{\omega_{x}^{2}+\omega_{y}^{2}}$. Hydrodynamic modes can be obtained by considering an ansatz of the 
form

$$
v=e^{i \omega t}\left(\alpha_{x} z \hat{x}+\alpha_{z} x \hat{z}\right)
$$

Substituting Eq. C4 in Eq. C1 gives the simultaneous equations

$$
\begin{aligned}
& \omega^{2} \alpha_{z}=\alpha_{x} \omega_{x}^{2}+\alpha_{z} \omega_{z}^{2} \\
& \omega^{2} \alpha_{x}=\alpha_{x} \omega_{x}^{2}+\alpha_{z} \omega_{z}^{2}
\end{aligned}
$$

One mode of interest for us is the $\omega=0$ mode since it has a velocity profile similar to Fig. 8 , This is what we call the Elliptic mode. If $\omega_{x}=\omega_{z}$, the mode looks like a rigid body rotation and can not exhibit viscous damping. For $\omega_{x} \neq \omega_{z}$ however we get a non-zero energy dissipation due to viscosity given by Eq. 10. The second mode of interest for us is what we call the Scissor mode which is well known in literature.

\section{Appendix D: Anisotropic viscosities in the relaxation time approximation}

In this section, we compute the anisotropic shear viscosities associated with the motion of a weakly interacting Fermi gas in the presence of an external potential in the relaxation time approximation [27]. For this section we explicitly keep $\hbar$ and $c$ in the expressions to ease comparisons with existing literature.

The Boltzmann equation in the relaxation time approximation is

$$
\frac{\partial f(x, p)}{\partial x^{\alpha}} V_{\alpha}+\frac{\partial f(x, p)}{\partial p^{\alpha}}\left(-\nabla_{\alpha} \phi\right)=-\frac{\delta f}{\tau}
$$

where $f$ is the distribution function, and $\tau$ is the effective relaxation time.

In equilibrium, the distribution function of occupied states for a weakly interacting gas is given by the Fermi-Dirac distribution function $f_{0}(x, p)=1 /\{\exp [(\epsilon(p)-p \cdot V(x)-\mu) / T(x)]+1\}$, where $\epsilon, p$ represent electron energy and momentum respectively. If a slowly varying local fluid velocity $V_{\alpha}(\alpha=1,2,3)$ is set up in the system, the electron distribution function is modified. To the lowest order in the derivatives of $V_{\alpha}$, we can write

$$
f(p)=f_{0}(\epsilon)+\delta f(p),
$$


where the non-equilibrium correction $\delta f(p)$ is of the form where

$$
\delta f(p)=-\left(\frac{\partial f_{0}}{\partial \mu}\right) v_{\alpha} p_{\beta} C_{\alpha \beta \gamma \delta}(\epsilon) V_{\gamma \delta}
$$

where $C_{\alpha \beta \gamma \delta}$ is a 4-rank tensor, $\mu$ represents the electron chemical potential, $v_{\alpha}=d \epsilon / d p_{\alpha}$ denotes the electron velocity, and $V_{\alpha \beta}$ is proportional to the derivative of the macroscopic fluid velocity defined as follows

$$
V_{\alpha \beta}=\frac{1}{2}\left(\frac{\partial V_{\alpha}}{\partial x_{\beta}}+\frac{\partial V_{\beta}}{\partial x_{\alpha}}\right)
$$

Similarly, in the presence of a slowly varying external potential $\phi$, Eq. D2 holds with

$$
\delta f(p)=-\left(\frac{\partial f_{0}}{\partial \mu}\right) v_{\alpha} D_{\alpha \gamma}(\epsilon) \partial_{\gamma} \phi
$$

Here we consider both $\partial_{\alpha} \phi$ and $V_{\alpha \beta}$ non-zero, and hence $\delta f$ is the sum of Eq. D3 and Eq. D5. After canceling out the terms proportional to $D$ (which are related to conductivity) the linearized Boltzmann equation within the relaxation time approximation of the collision integral takes the form

$$
\left(\frac{\partial f_{0}}{\partial \mu}\right)\left(v_{\alpha} p_{\beta} \frac{\partial V_{\alpha}}{\partial x_{\beta}}-\frac{1}{3} v_{\alpha} p_{\alpha} \operatorname{div} V\right)=-\frac{\delta f}{\tau}+(\nabla \phi) \cdot \frac{\partial \delta f}{\partial p}
$$

in analogy with Eq. 2 of [27] for the magnetic field case,

$$
\left(\frac{\partial f_{0}}{\partial \mu}\right)\left(v_{\alpha} p_{\beta} \frac{\partial V_{\alpha}}{\partial x_{\beta}}-\frac{1}{3} v_{\alpha} p_{\alpha} \operatorname{div} V\right)=-\frac{\delta f}{\tau}+\frac{e}{c}(v \times B) \cdot \frac{\partial \delta f}{\partial p}
$$

For ease of calculation, let us decompose the $\nabla \phi$ term on the R.H.S of Boltzmann equation as

$$
\nabla \phi=\hat{p}(\hat{p} . \nabla \phi)+(\nabla \phi-\hat{p}(\hat{p} . \nabla \phi))=\hat{p}(\hat{p} \cdot \nabla \phi)+\hat{p} \times(\nabla \phi \times \hat{p})
$$

In what follows, it is useful to define a basis $\xi^{\prime}$ for the 8 dimensional non-commutative algebra for the 4-rank tensor $C_{\gamma \delta \mu \nu}$ built out of the Kroenecker delta, Levi-civita and the components of the unit vector along the direction $\nabla \phi \times \hat{p}$ denoted by $\hat{b}$. 
The basis $\xi_{1}^{\prime}-\xi_{8}^{\prime}$ is defined as

$$
\begin{aligned}
& \xi_{1_{\alpha \beta \gamma \delta}}^{\prime}=\delta_{\alpha \gamma} \delta_{\beta \delta}+\delta_{\alpha \delta} \delta_{\beta \gamma} \\
& \xi_{2_{\alpha \beta \gamma \delta}}^{\prime}=\delta_{\alpha \beta} \delta_{\gamma \delta} \\
& \xi_{3_{\alpha \beta \gamma \delta}}^{\prime}=\hat{b}_{\alpha} \hat{b}_{\delta} \delta_{\beta \gamma}+\hat{b}_{\alpha} \hat{b}_{\gamma} \delta_{\beta \delta}+\delta_{\alpha \gamma} \hat{b}_{\beta} \hat{b}_{\delta}+\delta_{\alpha \delta} \hat{b}_{\beta} \hat{b}_{\gamma} \\
& \xi_{4_{\alpha \beta \gamma \delta}}^{\prime}=\delta_{\alpha \beta} \hat{b}_{\gamma} \hat{b}_{\delta} \\
& \xi_{5_{\alpha \beta \gamma \delta}}^{\prime}=\hat{b}_{\beta} \hat{b}_{\delta} \delta_{\gamma \delta} \\
& \xi_{6_{\alpha \beta \gamma \delta}}^{\prime}=\hat{b}_{\alpha} \hat{b}_{\beta} \hat{b}_{\gamma} \hat{b}_{\delta} \\
& \xi_{7_{\alpha \beta \gamma \delta}}^{\prime}=\delta_{\alpha \gamma} \hat{b}_{\beta \delta}+\hat{b}_{\alpha \gamma} \delta_{\beta \delta}+\delta_{\alpha \delta} \hat{b}_{\beta \gamma}+\hat{b}_{\alpha \delta} \delta_{\beta \gamma} \\
& \xi_{8_{\alpha \beta \gamma \delta}}^{\prime}=\hat{b}_{\alpha} \hat{b}_{\beta \gamma} \hat{b}_{\delta}+\hat{b}_{\alpha} \hat{b}_{\beta \delta} \hat{b}_{\gamma}+\hat{b}_{\alpha \gamma} \hat{b}_{\beta} \hat{b}_{\delta}+\hat{b}_{\alpha \delta} \hat{b}_{\beta} \hat{b}_{\gamma}
\end{aligned}
$$

Let us now simplify the L.H.S of Eq. D6

$$
\begin{aligned}
& \left(\frac{\partial f_{0}}{\partial \mu}\right)\left(v_{\alpha} p_{\beta} \frac{\partial V_{\alpha}}{\partial x_{\beta}}-\frac{1}{3} v_{\alpha} p_{\alpha} \operatorname{div} V\right) \\
& =\left(\frac{\partial f_{0}}{\partial \mu}\right) v_{\alpha} p_{\beta} V_{\mu \nu} \frac{1}{2}\left(\xi_{1_{\alpha \beta \mu \nu}^{\prime}}^{\prime}-\frac{2}{3} \xi_{2_{\alpha \beta \mu \nu}^{\prime}}\right)
\end{aligned}
$$

Similarly the R.H.S of Eq. D6 can be simplified as follows-

$$
\begin{aligned}
\text { R.H.S } & =-\frac{\delta f}{\tau}+(\nabla \phi)_{\alpha} \frac{\partial \delta f}{\partial p_{\alpha}}=-\frac{\delta f}{\tau}+(\hat{p}(\hat{p} . \nabla \phi)+\hat{p} \times(\nabla \phi \times \hat{p}))_{\alpha} \frac{\partial \delta f}{\partial p_{\alpha}} \\
& =-\delta f\left(\frac{1}{\tau}-\left(\frac{\hat{p} . \nabla \phi}{p}\right)\right)-(\hat{p} \times(\nabla \phi \times \hat{p}))_{\alpha} v_{a} C_{a \alpha \gamma \delta} V_{\gamma \delta}\left(\frac{\partial f_{0}}{\partial \mu}\right)
\end{aligned}
$$

Taking $\tau$ to the L.H.S we get

$$
\begin{aligned}
\tau \text { L.H.S } & =-\delta f\left(1-\tau\left(\frac{\hat{p} \cdot \nabla \phi}{p}\right)\right)-\tau(\hat{p} \times(\nabla \phi \times \hat{p}))_{\alpha} v_{a} C_{a \alpha \gamma \delta} V_{\gamma \delta}\left(\frac{\partial f_{0}}{\partial \mu}\right) \\
& =v_{\alpha} p_{\beta} V_{r s}\left(\frac{\partial f_{0}}{\partial \mu}\right)\left(C_{\alpha \beta r s}\left(1-\tau \frac{\hat{p} . \nabla \phi}{p}\right)-\frac{\tau b}{p} \epsilon_{\theta \beta \gamma} \hat{b}_{\gamma} C_{\alpha \theta r s}\right)
\end{aligned}
$$

where $b$ denotes the magnitude of the vector $\nabla \phi \times \hat{p}$.

Let $a=\left(1-\tau \frac{\hat{p} . \nabla \phi}{p}\right)$ and $x=\frac{\tau b}{p}$. If we denote the angle between $\nabla \phi$ and $\hat{p}$ as $\theta$, then $a=1-\frac{\nabla \phi \tau}{p} \cos \theta$ and $x=\frac{\tau \nabla \phi}{p} \sin \theta$.

Hence we get

$$
\tau \text { L.H.S }=v_{\alpha} p_{\beta} V_{r s}\left(\frac{\partial f_{0}}{\partial \mu}\right)\left(a C_{\alpha \beta r s}-x \epsilon_{\theta \beta \gamma} \hat{b}_{\gamma} C_{\alpha \theta r s}\right)
$$


Symmetrizing in $\alpha$ and $\beta$, we get

$$
\begin{aligned}
\tau \text { L.H.S } & =v_{\alpha} p_{\beta} V_{r s}\left(\frac{\partial f_{0}}{\partial \mu}\right)\left(a \frac{C_{\alpha \beta r s}+C_{\beta \alpha r s}}{2}-x \frac{\epsilon_{\theta \beta \gamma} \hat{b}_{\gamma} C_{\alpha \theta r s}+\epsilon_{\theta \alpha \gamma} \hat{b}_{\gamma} C_{\beta \theta r s}}{2}\right) \\
& =v_{\alpha} p_{\beta} V_{r s} \frac{1}{2} C_{\gamma \delta r s}\left(\frac{\partial f_{0}}{\partial \mu}\right)\left(a \delta_{\alpha \gamma} \delta_{\beta \delta}+a \delta_{\beta \gamma} \delta_{\alpha \delta}+x\left(b_{\beta \delta} \delta_{\gamma \alpha}+b_{\alpha \delta} \delta_{\gamma \beta}\right)\right)
\end{aligned}
$$

Subtracting the trace in $\alpha \beta$, we get

$$
\begin{aligned}
\tau \text { L.H.S } & =v_{\alpha} p_{\beta} V_{r s} \frac{1}{2} C_{\gamma \delta r s}\left(\frac{\partial f_{0}}{\partial \mu}\right)\left(a \delta_{\alpha \gamma} \delta_{\beta \delta}+a \delta_{\beta \gamma} \delta_{\alpha \delta}-\frac{2}{3} a \delta_{\gamma \delta} \delta_{\alpha \beta}+x\left(b_{\beta \delta} \delta_{\gamma \alpha}+b_{\alpha \delta} \delta_{\gamma \beta}+b_{\alpha \gamma} \delta_{\beta \delta}+b_{\beta \gamma} \delta_{\alpha \delta}\right)\right) \\
& =v_{\alpha} p_{\beta} V_{r s} \frac{1}{2} C_{\gamma \delta r s}\left(\frac{\partial f_{0}}{\partial \mu}\right)\left(a \xi_{1}^{\prime}-\frac{2}{3} a \xi_{2}^{\prime}+x \xi_{7}^{\prime}\right)_{\alpha \beta \gamma \delta}
\end{aligned}
$$

Now combining L.H.S and R.H.S we finally get

$$
\begin{aligned}
& \tau\left(\frac{\partial f_{0}}{\partial \mu}\right) v_{\alpha} p_{\beta} V_{\mu \nu} \frac{1}{2}\left(\xi_{1_{\alpha \beta \mu \nu}^{\prime}}^{\prime}-\frac{2}{3} \xi_{2_{\alpha \beta \mu \nu}^{\prime}}\right)=v_{\alpha} p_{\beta} V_{r s} \frac{1}{2} C_{\gamma \delta r s}\left(\frac{\partial f_{0}}{\partial \mu}\right)\left(a \xi_{1}^{\prime}-\frac{2}{3} a \xi_{2}^{\prime}+x \xi_{7}^{\prime}\right)_{\alpha \beta \gamma \delta} \\
& \Rightarrow \tau\left(\xi_{1_{\alpha \beta \mu \nu}^{\prime}}^{\prime}-\frac{2}{3} \xi_{2_{\alpha \beta \mu \nu}^{\prime}}\right)=\left(a \xi_{1}^{\prime}-\frac{2}{3} a \xi_{2}^{\prime}+x \xi_{7}^{\prime}\right)_{\alpha \beta \gamma \delta} C_{\gamma \delta \mu \nu}
\end{aligned}
$$

Writing $C_{\gamma \delta \mu \nu}=\left(\sum_{i=1}^{8} c_{i} \xi_{i \gamma \delta \mu \nu}^{\prime}\right)$ we can now solve for the coefficients

$$
\begin{aligned}
& c_{1}=\frac{a \tau}{2\left(a^{2}+4 x^{2}\right)}, c_{2}=-\frac{\tau\left(a^{2}-2 x^{2}\right)}{3 a\left(a^{2}+4 x^{2}\right)}, c_{3}=\frac{3 a \tau x^{2}}{2\left(a^{2}+x^{2}\right)\left(a^{2}+4 x^{2}\right)}, c_{4}=c_{5}=-\frac{2 \tau x^{2}}{a\left(a^{2}+4 x^{2}\right)}, \\
& c_{6}=\frac{6 \tau x^{4}}{a\left(a^{2}+x^{2}\right)\left(a^{2}+4 x^{2}\right)}, c_{7}=-\frac{\tau x}{2\left(a^{2}+4 x^{2}\right)}, c_{8}=-\frac{3 \tau x^{3}}{2\left(a^{2}+x^{2}\right)\left(a^{2}+4 x^{2}\right)}
\end{aligned}
$$

The viscosity tensor is given as

$$
\eta_{\alpha \beta a b}=-\frac{2}{(2 \pi \hbar)^{3}} \int \mathrm{d}^{3} p\left(\frac{\partial f_{0}}{\partial \mu}\right) v_{\alpha} p_{\beta} v_{\gamma} p_{\delta}\left(\sum_{i=1}^{8} c_{i} \xi_{i \gamma \delta a b}^{\prime}\right) .
$$

It is convenient to decompose the tensor $\eta_{\alpha \beta a b}$ in to 5 irreducible components corresponding to 5 tensors $M_{i \alpha \beta a b}(i=0, \cdots 4)$ in a system with a special direction $\hat{E}=\nabla \phi /|\nabla \phi|$ and reflection symmetry.

$$
\eta_{\alpha \beta \gamma \delta}=\sum_{i=0}^{4} \eta_{i} M_{i \alpha \beta \gamma \delta}
$$


The tensors $M_{i}$ are

$$
\begin{aligned}
& M_{0}=3 \xi_{6}-\xi_{4}-\xi_{5}+\frac{\xi_{2}}{3} \\
& M_{1}=\xi_{1}-\xi_{2}-\xi_{3}+\xi_{4}+\xi_{5}+\xi_{6} \\
& M_{2}=\xi_{3}-4 \xi_{6} \\
& M_{3}=-\frac{1}{2}\left(\xi_{7}-\xi_{8}\right) \\
& M_{4}=-\xi_{8}
\end{aligned}
$$

where the basis $\xi_{1}-\xi_{8}$ is defined as

$$
\begin{aligned}
& \xi_{1_{\alpha \beta \gamma \delta}}=\delta_{\alpha \gamma} \delta_{\beta \delta}+\delta_{\alpha \delta} \delta_{\beta \gamma} \\
& \xi_{2_{\alpha \beta \gamma \delta}}=\delta_{\alpha \beta} \delta_{\gamma \delta} \\
& \xi_{3_{\alpha \beta \gamma \delta}}=\hat{E}_{\alpha} \hat{E}_{\delta} \delta_{\beta \gamma}+\hat{E}_{\alpha} \hat{E}_{\gamma} \delta_{\beta \delta}+\delta_{\alpha \gamma} \hat{E}_{\beta} \hat{E}_{\delta}+\delta_{\alpha \delta} \hat{E}_{\beta} \hat{E}_{\gamma} \\
& \xi_{4_{\alpha \beta \gamma \delta}}=\delta_{\alpha \beta} \hat{E}_{\gamma} \hat{E}_{\delta} \\
& \xi_{5_{\alpha \beta \gamma \delta}}=\hat{E}_{\beta} \hat{E}_{\delta} \delta_{\gamma \delta} \\
& \xi_{6_{\alpha \beta \gamma \delta}}=\hat{E}_{\alpha} \hat{E}_{\beta} \hat{E}_{\gamma} \hat{E}_{\delta} \\
& \xi_{7_{\alpha \beta \gamma \delta}}=\delta_{\alpha \gamma} \hat{E}_{\beta \delta}+\hat{E}_{\alpha \gamma} \delta_{\beta \delta}+\delta_{\alpha \delta} \hat{E}_{\beta \gamma}+\hat{E}_{\alpha \delta} \delta_{\beta \gamma} \\
& \xi_{8_{\alpha \beta \gamma \delta}}=\hat{E}_{\alpha} \hat{E}_{\beta \gamma} \hat{E}_{\delta}+\hat{E}_{\alpha} \hat{E}_{\beta \delta} \hat{E}_{\gamma}+\hat{E}_{\alpha \gamma} \hat{E}_{\beta} \hat{E}_{\delta}+\hat{E}_{\alpha \delta} \hat{E}_{\beta} \hat{E}_{\gamma},
\end{aligned}
$$

where $\hat{E}$ is the unit vector along the gradient of the potential.

The components $\eta_{i}$ can be extracted by projecting onto $M_{i}$ and performing the three dimensional momentum integral in Eq. D16. For arbitrarily large $\frac{|\tau \nabla \phi|}{k_{F}}$ the momentum integrals can not be performed analytically in general. However, we are interested in $\frac{|\tau \nabla \phi|}{k_{F}} \lesssim 1$, where the corrections to isotropy just start to become important. Then one can expand in $|\tau \nabla \phi|$ and perform the angular integrals to obtain,

$$
\begin{aligned}
& \eta_{0}=\eta(0)\left[1-\frac{31}{21} \tau^{2}(\nabla \phi)^{2} \frac{I_{2}}{I_{1}}+\mathcal{O}\left((\tau \nabla \phi)^{4}\right)\right] \\
& \eta_{1}=\eta(0)\left[1-\frac{13}{7} \tau^{2}(\nabla \phi)^{2} \frac{I_{2}}{I_{1}}+\mathcal{O}\left((\tau \nabla \phi)^{4}\right)\right] \\
& \eta_{2}=\eta(0)\left[1-\frac{11}{7} \tau^{2}(\nabla \phi)^{2} \frac{I_{2}}{I_{1}}+\mathcal{O}\left((\tau \nabla \phi)^{4}\right)\right] \\
& \eta_{3}=0, \eta_{4}=0
\end{aligned}
$$


where

$$
\eta(0)=\int p^{6} d p \frac{\tau}{15 \pi^{2} m^{2} \hbar^{3}}\left(\frac{\partial f_{0}}{\partial \mu}\right)
$$

is the shear viscosity in the absence of $\nabla \phi$, and $I_{1}$ and $I_{2}$ are.

$$
I_{1}=\int p^{6} d p\left(\frac{\partial f_{0}}{\partial \mu}\right), I_{2}=\int p^{4} d p\left(\frac{\partial f_{0}}{\partial \mu}\right)
$$

In particular, in the degenerate limit $(T \ll \mu)$

$$
\left(\frac{\partial f_{0}}{\partial \mu}\right) \approx \delta\left(\frac{p^{2}}{2 m}-\mu\right)
$$

and $\frac{I_{1}}{I_{2}} \approx \frac{1}{k_{F}^{2}}$ where $k_{F}=\left(3 \pi^{2} n\right)^{1 / 3}$ as before.

We can write Eq. D20 in the form Eq. 64 by relating the relaxation time $\tau$ to the mean free path $\lambda$

$$
\frac{\tau}{k_{F}}=\frac{\tau}{k_{F}} \frac{E_{F}}{E_{F}}=\frac{\lambda}{2 E_{F}}
$$

where we have used $E_{F} / k_{F}=v_{F} / 2$, and $\tau v_{F}=\lambda$ is the mean free path.

This gives,

$$
\begin{aligned}
& \eta_{0}=\eta(0)\left[1-\frac{31}{84} \frac{\lambda^{2}(\nabla \phi)^{2}}{\mu^{2}}+\mathcal{O}\left((\tau \nabla \phi)^{4}\right)\right] \\
& \eta_{1}=\eta(0)\left[1-\frac{13}{28} \frac{\lambda^{2}(\nabla \phi)^{2}}{\mu^{2}}+\mathcal{O}\left((\tau \nabla \phi)^{4}\right)\right] \\
& \eta_{2}=\eta(0)\left[1-\frac{11}{28} \frac{\lambda^{2}(\nabla \phi)^{2}}{\mu^{2}}+\mathcal{O}\left((\tau \nabla \phi)^{4}\right)\right] \\
& \eta_{3}=0, \eta_{4}=0
\end{aligned}
$$

where

$$
\eta(0)=\frac{(2 m \mu)^{\frac{5}{2}} \tau}{15 \pi^{2} \hbar^{3} m}
$$

in the degenerate limit.

Eq. D25 gives an explicit result of the calculation in the relaxation time approximation which shows that the correction to the viscosity has the form Eq. 64. Hearteningly, the sign of $c_{(i)}$ is negative, meaning that the viscosity is reduced due to the external potential, a feature found is 
strongly coupled theories where a quasi-particle description is not possible and hence the Boltzmann transport equation can not be used to calculate the viscosity.

Interestingly, in the degenerate limit it is possible to do the momentum integrals analytically for general $\nabla \phi$. Using $\left(\frac{\partial f_{0}}{\partial \mu}\right)=\delta\left(\frac{p^{2}}{2 m}-\mu\right)$, we get (here $\left.x=\frac{\nabla \phi \tau}{\sqrt{2 m \mu}}\right)$

$$
\begin{aligned}
& \eta_{0}=\frac{(2 m \mu)^{\frac{5}{2}} \tau}{96 m \hbar^{3} \pi^{2} x^{5} \sqrt{3 x^{2}+1}}\left[-8 \sqrt{3\left(x^{2}+1\right.}\left(5 x^{4}+18 x^{2}+9\right) \tanh ^{-1}(x)\right. \\
& -24 x \sqrt{3 x^{2}+1}\left(5 x^{2}+3\right)-6\left(8 x^{4}+11 x^{2}+3\right) \\
& \left.\log \left(\frac{x\left(7 x-4 \sqrt{3 x^{2}+1}\right)+1}{x\left(4 \sqrt{3 x^{2}+1}+7 x\right)+1}\right)\right] \\
& \eta_{1}=\frac{(2 m \mu)^{\frac{5}{2}} \tau}{96 m \hbar^{3} \pi^{2} x^{5} \sqrt{3 x^{2}+1}}\left[-4 x\left(x^{2}+3\right) \sqrt{3 x^{2}+1}\right. \\
& +4 \sqrt{3 x^{2}+1}\left(x^{4}-6 x^{2}-3\right) \tanh ^{-1}(x)-\left(3+4 x^{4}+9 x^{2}\right) \\
& \left.\log \left(\frac{x\left(7 x-4 \sqrt{3 x^{2}+1}\right)+1}{x\left(4 \sqrt{3 x^{2}+1}+7 x\right)+1}\right)\right] \\
& \eta_{2}=\frac{(2 m \mu)^{\frac{5}{2}} \tau}{48 m \hbar^{3} \pi^{2} x^{5} \sqrt{3 x^{2}+1}}\left[8 x \sqrt{3 x^{2}+1}\left(4 x^{2}+3\right)\right. \\
& +4 \sqrt{3 x^{2}+1}\left(x^{4}+6 x^{2}+3\right) \tanh ^{-1}\left(\frac{2 x}{x^{2}+1}\right)+\left(6+13 x^{4}+21 x^{2}\right) \\
& \left.\log \left(\frac{x\left(7 x-4 \sqrt{3 x^{2}+1}\right)+1}{x\left(4 \sqrt{3 x^{2}+1}+7 x\right)+1}\right)\right] \\
& \eta_{3}=0 \\
& \eta_{4}=0
\end{aligned}
$$


Expanding in small $x$ we obtain,

$$
\begin{aligned}
& \eta_{0}=\frac{(2 m \mu)^{\frac{5}{2}} \tau}{15 \pi^{2} \hbar^{3} m}\left(1-\frac{31 \tau^{2} \nabla \phi^{2}}{42 m \mu}+\mathcal{O}\left[\left(\frac{\tau \nabla \phi}{\sqrt{2 m \mu}}\right)^{4}\right]\right), \quad \eta_{1}=\frac{(2 m \mu)^{\frac{5}{2}} \tau}{15 \pi^{2} \hbar^{3} m}\left(1-\frac{13 \tau^{2} \nabla \phi^{2}}{14 m \mu}+\mathcal{O}\left[\left(\frac{\tau \nabla \phi}{\sqrt{2 m \mu}}\right)^{4}\right]\right), \\
& \eta_{2}=\frac{(2 m \mu)^{\frac{5}{2}} \tau}{15 \pi^{2} \hbar^{3} m}\left(1-\frac{11 \tau^{2} \nabla \phi^{2}}{14 m \mu}+\mathcal{O}\left[\left(\frac{\tau \nabla \phi}{\sqrt{2 m \mu}}\right)^{4}\right]\right), \\
& \eta_{3}=0, \quad \eta_{4}=0 .
\end{aligned}
$$

[1] A. Adams, L. D. Carr, T. Schäfer, P. Steinberg, and J. E. Thomas, "Strongly Correlated Quantum Fluids: Ultracold Quantum Gases, Quantum Chromodynamic Plasmas, and Holographic Duality," New J. Phys., vol. 14, p. 115009, 2012.

[2] A. Bulgac, M. M. Forbes, and P. Magierski, "The Unitary Fermi Gas: From Monte Carlo to Density Functionals," Lect. Notes Phys., vol. 836, pp. 305-373, 2012.

[3] R. S. Bhalerao, "Transport properties of the fluid produced at RHIC," Pramana, vol. 75, pp. 247-258, 2010.

[4] D. Page and S. Reddy, "Dense Matter in Compact Stars: Theoretical Developments and Observational Constraints," Ann. Rev. Nucl. Part. Sci., vol. 56, pp. 327-374, 2006.

[5] M. G. Alford, H. Nishimura, and A. Sedrakian, "Transport coefficients of two-flavor superconducting quark matter," Phys. Rev., vol. C90, no. 5, p. 055205, 2014.

[6] A. Gezerlis and J. Carlson, "Strongly paired fermions: Cold atoms and neutron matter," Phys. Rev., vol. C77, p. 032801, 2008.

[7] R. V. Gavai, S. Gupta, and S. Mukherjee, "A New method for computation of QCD thermodynamics: EOS, specific heat and speed of sound," PoS, vol. LAT2005, p. 173, 2006.

[8] G. Wlazlowski, P. Magierski, and J. E. Drut, "Shear Viscosity of a Unitary Fermi Gas," Phys. Rev. Lett., vol. 109, p. 020406, 2012.

[9] G. Wlazowski, W. Quan, and A. Bulgac, "Perfect-fluid behavior of a dilute Fermi gas near unitary," Phys. Rev., vol. A92, no. 6, p. 063628, 2015.

[10] H. B. Meyer, "Transport properties of the quark-gluon plasma from lattice qcd," Nuclear Physics A, vol. 830, no. 1, pp. 641c-648c, 2009.

[11] J. Maldacena, "The large-n limit of superconformal field theories and supergravity," International journal of theoretical physics, vol. 38, no. 4, pp. 1113-1133, 1999.

[12] D. T. Son and A. O. Starinets, "Minkowski space correlators in AdS / CFT correspondence: Recipe and applications," JHEP, vol. 0209, p. 042, 2002.

[13] P. K. Kovtun, D. T. Son, and A. O. Starinets, "Viscosity in strongly interacting quantum field theories from black hole physics," Phys. Rev. Lett., vol. 94, p. 111601, Mar 2005.

[14] M. Brigante, H. Liu, R. C. Myers, S. Shenker, and S. Yaida, "Viscosity Bound Violation in Higher 
Derivative Gravity," Phys.Rev., vol. D77, p. 126006, 2008.

[15] M. Brigante, H. Liu, R. C. Myers, S. Shenker, and S. Yaida, "The Viscosity Bound and Causality Violation," Phys.Rev.Lett., vol. 100, p. 191601, 2008.

[16] Y. Kats and P. Petrov, "Effect of curvature squared corrections in AdS on the viscosity of the dual gauge theory," JHEP, vol. 0901, p. 044, 2009.

[17] A. Buchel, R. C. Myers, and A. Sinha, "Beyond eta/s = 1/4 pi," JHEP, vol. 0903, p. $084,2009$.

[18] A. Sinha and R. C. Myers, "The Viscosity bound in string theory," Nucl.Phys., vol. A830, pp. 295C$298 \mathrm{C}, 2009$.

[19] S. Cremonini, "The Shear Viscosity to Entropy Ratio: A Status Report," Mod.Phys.Lett., vol. B25, pp. 1867-1888, 2011.

[20] U. Heinz and R. Snellings, "Collective flow and viscosity in relativistic heavy-ion collisions," Ann. Rev. Nucl. Part. Sci., vol. 63, pp. 123-151, 2013.

[21] T. Schäfer, "The Shear Viscosity to Entropy Density Ratio of Trapped Fermions in the Unitarity Limit," Phys. Rev., vol. A76, p. 063618, 2007.

[22] C. Cao, E. Elliott, J. Joseph, H. Wu, J. Petricka, T. Schäfer, and J. E. Thomas, "Universal quantum viscosity in a unitary fermi gas," Science, vol. 331, no. 6013, pp. 58-61, 2011.

[23] J. A. Joseph, E. Elliott, and J. E. Thomas, "Shear viscosity of a unitary fermi gas near the superfluid phase transition," Phys. Rev. Lett., vol. 115, p. 020401, Jul 2015.

[24] M. Martinez, R. Ryblewski, and M. Strickland, "Boost-Invariant (2+1)-dimensional Anisotropic Hydrodynamics," Phys. Rev., vol. C85, p. 064913, 2012.

[25] L. D. Landau and E. M. Lifshitz, "Fluid Mechanics, Second Edition: Volume 6 (Course of Theoretical Physics)," Butterworth-Heinemann, 2 ed., Jan. 1987.

[26] K. Tuchin, "On viscous flow and azimuthal anisotropy of quark-gluon plasma in strong magnetic field," J. Phys., vol. G39, p. 025010, 2012.

[27] D. D. Ofengeim and D. G. Yakovlev, "Shear viscosity in magnetized neutron star crust," Europhys. Lett., vol. 112, no. 5, p. 59001, 2015.

[28] K. Landsteiner and J. Mas, "The Shear viscosity of the non-commutative plasma," JHEP, vol. 0707, p. $088,2007$.

[29] T. Azeyanagi, W. Li, and T. Takayanagi, "On String Theory Duals of Lifshitz-like Fixed Points," JHEP, vol. 0906, p. 084, 2009.

[30] M. Natsuume and M. Ohta, "The Shear viscosity of holographic superfluids," Prog.Theor.Phys., vol. 124, pp. 931-951, 2010.

[31] J. Erdmenger, P. Kerner, and H. Zeller, "Non-universal shear viscosity from Einstein gravity," Phys.Lett., vol. B699, pp. 301-304, 2011.

[32] P. Basu and J.-H. Oh, "Analytic Approaches to Anisotropic Holographic Superfluids," JHEP, vol. 1207, p. 106, 2012.

[33] J. Erdmenger, P. Kerner, and H. Zeller, "Transport in Anisotropic Superfluids: A Holographic Descrip- 
tion," JHEP, vol. 1201, p. 059, 2012.

[34] D. Mateos and D. Trancanelli, "The anisotropic N=4 super Yang-Mills plasma and its instabilities," Phys.Rev.Lett., vol. 107, p. 101601, 2011.

[35] D. Mateos and D. Trancanelli, "Thermodynamics and Instabilities of a Strongly Coupled Anisotropic Plasma," JHEP, vol. 1107, p. 054, 2011.

[36] N. Iizuka and K. Maeda, "Study of Anisotropic Black Branes in Asymptotically anti-de Sitter," JHEP, vol. 1207, p. 129, 2012.

[37] A. Rebhan and D. Steineder, "Violation of the Holographic Viscosity Bound in a Strongly Coupled Anisotropic Plasma," Phys.Rev.Lett., vol. 108, p. 021601, 2012.

[38] J. Polchinski and E. Silverstein, "Large-density field theory, viscosity, and ' $2 k_{F}$ ' singularities from string duals," Class.Quant.Grav., vol. 29, p. 194008, 2012.

[39] D. Giataganas, "Probing strongly coupled anisotropic plasma," JHEP, vol. 07, p. 031, 2012.

[40] K. A. Mamo, "Holographic RG flow of the shear viscosity to entropy density ratio in strongly coupled anisotropic plasma," JHEP, vol. 1210, p. 070, 2012.

[41] A. Bhattacharyya and D. Roychowdhury, "Viscosity bound for anisotropic superfluids in higher derivative gravity," JHEP, vol. 03, p. 063, 2015.

[42] S. Jain, N. Kundu, K. Sen, A. Sinha, and S. P. Trivedi, "A Strongly Coupled Anisotropic Fluid From Dilaton Driven Holography," JHEP, vol. 01, p. 005, 2015.

[43] R. Critelli, S. Finazzo, M. Zaniboni, and J. Noronha, "Anisotropic shear viscosity of a strongly coupled non-Abelian plasma from magnetic branes," Phys.Rev., vol. D90, no. 6, p. 066006, 2014.

[44] X.-H. Ge, Y. Ling, C. Niu, and S.-J. Sin, "Thermoelectric conductivities, shear viscosity, and stability in an anisotropic linear axion model," Phys. Rev., vol. D92, no. 10, p. 106005, 2015.

[45] S. Jain, R. Samanta, and S. P. Trivedi, "The Shear Viscosity in Anisotropic Phases," JHEP, vol. 10, p. 028, 2015.

[46] W. Ketterle and M. W. Zwierlein, "Making, probing and understanding ultracold fermi gases," arXiv preprint arXiv:0801.2500, 2008.

[47] D. Guéry-Odelin and S. Stringari, "Scissors mode and superfluidity of a trapped bose-einstein condensed gas," Phys. Rev. Lett., vol. 83, pp. 4452-4455, Nov 1999.

[48] O. M. Maragò, S. A. Hopkins, J. Arlt, E. Hodby, G. Hechenblaikner, and C. J. Foot, "Observation of the scissors mode and evidence for superfluidity of a trapped bose-einstein condensed gas," Phys. Rev. Lett., vol. 84, pp. 2056-2059, Mar 2000.

[49] M. J. Wright, S. Riedl, A. Altmeyer, C. Kohstall, E. R. S. Guajardo, J. H. Denschlag, and R. Grimm, "Finite-temperature collective dynamics of a fermi gas in the bec-bcs crossover," Phys. Rev. Lett., vol. 99, p. 150403, Oct 2007.

[50] S. Giorgini, L. P. Pitaevskii, and S. Stringari, "Theory of ultracold atomic fermi gases," Rev. Mod. Phys., vol. 80, pp. 1215-1274, Oct 2008.

[51] D. T. Son, "Vanishing bulk viscosities and conformal invariance of the unitary fermi gas," Phys. Rev. 
Lett., vol. 98, p. 020604, Jan 2007.

[52] E. Taylor and M. Randeria, "Viscosity of strongly interacting quantum fluids: Spectral functions and sum rules," Phys. Rev. A, vol. 81, p. 053610, May 2010.

[53] L. D. Landau and E. M. Lifshitz, "Fluid Mechanics, First Edition: Volume 10 (Course of Theoretical Physics)," Pergamon Press, 1981.

[54] M. Randeria, W. Zwerger, and M. Zwierlein, "The bcsbec crossover and the unitary fermi gas," in The BCS-BEC Crossover and the Unitary Fermi Gas (W. Zwerger, ed.), vol. 836 of Lecture Notes in Physics, pp. 1-32, Springer Berlin Heidelberg, 2012.

[55] G. M. Bruun and H. Smith, "Viscosity and thermal relaxation for a resonantly interacting fermi gas," Phys. Rev. A, vol. 72, p. 043605, Oct 2005.

[56] G. M. Bruun and H. Smith, "Shear viscosity and damping for a Fermi gas in the unitarity limit," Phys. Rev., vol. A75, p. 043612, 2007.

[57] M. Bluhm and T. Schäfer, "Dissipative fluid dynamics for the dilute Fermi gas at unitarity: Anisotropic fluid dynamics," Phys. Rev., vol. A92, no. 4, p. 043602, 2015.

[58] P. Massignan, G. M. Bruun, and H. Smith, "Viscous relaxation and collective oscillations in a trapped fermi gas near the unitarity limit," Phys. Rev. A, vol. 71, p. 033607, Mar 2005.

[59] G. M. Bruun and H. Smith, "Frequency and damping of the scissors mode of a fermi gas," Phys. Rev. A, vol. 76, p. 045602, Oct 2007.

[60] T. Schäfer, "Generalized theory of diffusion based on kinetic theory," Phys. Rev. A, vol. 94, p. 043644, Oct 2016 .

[61] S. Riedl, E. R. Sánchez Guajardo, C. Kohstall, A. Altmeyer, M. J. Wright, J. H. Denschlag, R. Grimm, G. M. Bruun, and H. Smith, "Collective oscillations of a fermi gas in the unitarity limit: Temperature effects and the role of pair correlations," Phys. Rev. A, vol. 78, p. 053609, Nov 2008.

[62] M. Bluhm and T. Schäfer, "Model-independent determination of the shear viscosity of a trapped unitary fermi gas: Application to high-temperature data," Phys. Rev. Lett., vol. 116, p. 115301, Mar 2016.

[63] M. J. H. Ku, A. T. Sommer, L. W. Cheuk, and M. W. Zwierlein, "Revealing the superfluid lambda transition in the universal thermodynamics of a unitary fermi gas," Science, vol. 335, no. 6068, pp. 563$567,2012$.

[64] G. Rupak and T. Schäfer, "Shear viscosity of a superfluid fermi gas in the unitarity limit," Phys. Rev. $A$, vol. 76, p. 053607, Nov 2007.

[65] H. Guo, D. Wulin, C.-C. Chien, and K. Levin, "Microscopic Approach to Shear Viscosities in Superfluid Gases: From BCS to BEC," Phys. Rev. Lett., vol. 107, p. 020403, 2011.

[66] T. Enss, R. Haussmann, and W. Zwerger, "Viscosity and scale invariance in the unitary Fermi gas," Annals Phys., vol. 326, pp. 770-796, 2011.

[67] A. Bulgac, J. E. Drut, and P. Magierski, "Thermodynamics of a trapped unitary fermi gas," Phys. Rev. Lett., vol. 99, p. 120401, Sep 2007.

[68] M. M. Forbes, S. Gandolfi, and A. Gezerlis, "Resonantly Interacting Fermions In a Box," Phys. Rev. 
Lett., vol. 106, p. 235303, 2011.

[69] R. Haussmann, "Properties of a fermi liquid at the superfluid transition in the crossover region between bcs superconductivity and bose-einstein condensation," Phys. Rev. B, vol. 49, pp. 12975-12983, May 1994.

[70] O. Goulko and M. Wingate, "Numerical study of the unitary fermi gas across the superfluid transition," Phys. Rev. A, vol. 93, p. 053604, May 2016.

[71] E. Burovski, N. Prokof'ev, B. Svistunov, and M. Troyer, "Critical temperature and thermodynamics of attractive fermions at unitarity," Phys. Rev. Lett., vol. 96, p. 160402, Apr 2006.

[72] A. Bulgac, J. E. Drut, and P. Magierski, "Spin 1/2 fermions in the unitary regime: A superfluid of a new type," Phys. Rev. Lett., vol. 96, p. 090404, Mar 2006.

[73] R. Haussmann, W. Rantner, S. Cerrito, and W. Zwerger, "Thermodynamics of the bcs-bec crossover," Phys. Rev. A, vol. 75, p. 023610, Feb 2007.

[74] C. Cao, "Section 6.2 of phd thesis - universal quantum viscosity in a unitary fermi gas,"

[75] M. W. Zwierlein, J. R. Abo-Shaeer, A. Schirotzek, C. H. Schunck, and W. Ketterle, "Vortices and superfluidity in a strongly interacting Fermi gas," Nature (London), vol. 435, pp. 1047-1051, June 2005.

[76] D. T. Son and M. Wingate, "General coordinate invariance and conformal invariance in nonrelativistic physics: Unitary Fermi gas," Annals Phys., vol. 321, pp. 197-224, 2006.

[77] M. M. Forbes, "The Unitary Fermi Gas in a Harmonic Trap and its Static Response," 2012, arXiv 1211.3779.

[78] M. Goossens, R. Erdélyi, and M. S. Ruderman, "Resonant mhd waves in the solar atmosphere," Space Science Reviews, vol. 158, no. 2, pp. 289-338, 2010.

[79] X. Bekaert, E. Meunier, and S. Moroz, "Towards a gravity dual of the unitary Fermi gas," Phys. Rev., vol. D85, p. 106001, 2012.

[80] P. Vignolo and A. Minguzzi, "Shell structure in the density profiles for noninteracting fermions in anisotropic harmonic confinement," Phys. Rev. A, vol. 67, p. 053601, May 2003.

[81] E. Vogt, M. Feld, B. Fröhlich, D. Pertot, M. Koschorreck, and M. Köhl, "Scale invariance and viscosity of a two-dimensional fermi gas," Phys. Rev. Lett., vol. 108, p. 070404, Feb 2012.

[82] S. Chakraborty and R. Samanta, "Viscosity for anisotropic reissner-nordström black branes," Phys. Rev. D, vol. 95, p. 106012, May 2017.

[83] H. Heiselberg, "Collective Modes of Trapped Gases at the BEC-BCS Crossover," Phys. Rev. Lett., vol. 93, p. 040402, 2004. 